\title{
Determination of the electric field and its Hilbert transform in femtosecond electro-optic sampling
}

\author{
P. Sulzer $\odot,{ }^{1}$ K. Oguchi,${ }^{1}$ J. Huster, ${ }^{1}$ M. Kizmann $\odot,{ }^{1}$ T. L. M. Guedes, ${ }^{1}$ A. Liehl $\odot,{ }^{1}$ C. Beckh, ${ }^{1}$ A. S. Moskalenko $\odot,{ }^{1,2}$ \\ G. Burkard ${ }^{1},{ }^{1}$ D. V. Seletskiy ${ }^{(1,},{ }^{1,3}$ and A. Leitenstorfer ${ }^{1}{ }^{1, *}$ \\ ${ }^{1}$ Department of Physics and Center for Applied Photonics, University of Konstanz, D-78457 Konstanz, Germany \\ ${ }^{2}$ Department of Physics, KAIST, Daejeon 34141, Republic of Korea \\ ${ }^{3}$ Department of Engineering Physics, Polytechnique Montréal, Montréal, Canada H3T 1J4
}

(Received 26 November 2019; accepted 4 February 2020; published 16 March 2020)

\begin{abstract}
We demonstrate time-domain sampling of mid-infrared electric field transients and their conjugate counterparts exploiting the dynamical Pockels effect. To this end, the complete polarization change of few-femtosecond probe pulses is studied. An intuitive picture based on a phasor representation is established before gaining quantitative understanding in experiment and theory. In the standard version of electro-optic sampling, the electric field is determined by analyzing the change of ellipticity of the probe polarization. Beyond this, we find that a temporal gradient of the input electric field manifests itself in a rotation of the polarization ellipsoid of the probe. The relative contribution of sum- and difference-frequency mixing processes and their spectral distribution over the near-infrared probe bandwidth are identified as key aspects. If one of these processes dominates, detecting ellipticity changes and polarization rotation as a function of time delay results in two wave forms which are Hilbert transforms of each other. Such conditions may be achieved by angle phase matching in birefringent materials or spectral filtering of the probe after the nonlinear interaction. In this case, a static phase introduced by birefringence or reflection at metallic mirrors results in a specific phase shift of both time traces with respect to the input electric field. Contributions from sum- and difference-frequency generation are found to be equivalent when using electro-optic sensors with isotropic refractive index. Polarization rotations in the lowand high-frequency parts of the probe then tend to cancel out. In this limit, spurious additional phase shifts do not change the phase of the detected transients. This fact leads to a robust recovery of the carrier-envelope phase of the input wave form. Clarifying the role of imperfections of superachromatic phase retarders completes our survey on proper determination of the electric field and its conjugate variable.
\end{abstract}

DOI: 10.1103/PhysRevA.101.033821

\section{INTRODUCTION}

Free-space electro-optic sampling (EOS) with femtosecond probe pulses represents a powerful tool for direct characterization of phase-locked electromagnetic transients in the time domain. Initially applied to far-infrared radiation in the few-terahertz range [1], the bandwidth has been extended over the last decades to cover the entire mid-infrared and parts of the near-infrared spectral range [2-7]. The technique represents an important cornerstone for studying the dynamics of low-frequency degrees of freedom of matter with subcycle temporal resolution, providing access to the full polarization response in amplitude and phase [8-13]. In addition to analyzing the average or coherent part of the electric field, recent years have seen a push to employ EOS for time-domain studies of quantum fluctuations via statistical readout. The direct observation of the vacuum noise of the electromagnetic field [14,15] represents an early breakthrough in this context. Subsequently, noise changes synchronized to coherent multiterahertz transients were found and interpreted as evidence for squeezed states [16]. Very recently, exploitation of spatiotemporal noise correlations has allowed characterizing the amplitude response of electro-optic detection using either

\footnotetext{
*alfred.leitenstorfer@uni-konstanz.de
}

thermal fields or even the bare vacuum fluctuations as an input [17]. Various prospects for interesting applications of this subcycle quantum technology have been worked out theoretically [18-21]. These studies include the investigation of traces of ultrashort pulsed squeezed states and their physical implications [18,19], as well as ultrastrong light-matter coupling [20] or manipulation of the electromagnetic groundstate fluctuations through reflecting plates [21].

Overall, quantum optics has been extremely successful in analyzing the quantum nature of light based on homodyning and photon correlation measurements [22,23]. Following from Heisenberg's uncertainty principle, conjugate variables represent a central concept in this area. It has been pointed out for the single-mode case with a well-defined frequency that the electric field and its temporal derivative are conjugate variables, although normalization by the frequency has been applied already in this work [24]. In the case of multimode fields extending over a bandwidth similar to their center frequency, it was recently suggested that the Hilbert transform of the electric field is a proper choice for the conjugate variable [25]. Mathematically, this quantity is obtained as follows [26]: In a Fourier transform of the original field trace, each frequency component is phase shifted by $\pi / 2$, thus retaining an identical amplitude spectrum. The result is then brought back into the time domain by an inverse Fourier transform. Note that in contrast to the temporal derivative of 
a transient, the Hilbert transform is nonlocal in time, which in principle necessitates knowledge about both past and future amplitudes of a wave packet [25]. Consequently, the question arises as to whether also the temporal derivative and/or the Hilbert transform may be measured experimentally by EOS, thereby extending this technique towards a full quantum tomography of light in the time domain.

In this work, we show that EOS is readily able to provide this information: monitoring not only the change in polarization ellipticity but also the rotation of linear polarization induced by the nonlinear mixing between the ultrashort probe pulses and a multiterahertz field indeed gives access to its Hilbert transform, depending on the precise experimental arrangement. Based on the frequency-domain description of EOS [27], we develop an intuitive picture explaining how the signals arise. The balance between sum and differencefrequency processes as well as the relative phase between newly generated and original probe photons are identified as the critical parameters in Sec. II. Consecutively, we demonstrate the validity of the model both experimentally and theoretically by closely examining electro-optic signals as a function of the involved probe frequencies. The cases of electro-optic detection with phase matching based on birefringent crystals and with optically isotropic media are investigated in Secs. III A and III B, respectively. In this context, the relative phase turns out to represent a close analogy to the local oscillator phase in standard homodyning experiments. We then provide rigorous analytical and numerical calculations in Sec. IV, supporting the qualitative model developed in Sec. II and quantitatively explaining the experimental results in Sec. III. This understanding enables us to show how the two quadratures may be measured simultaneously, as desired in any kind of quantum tomography.

\section{EXPERIMENTAL SETUP AND INTUITIVE FREQUENCY-DOMAIN PICTURE}

We now explain the experimental setup for electro-optic detection of multiterahertz electric fields. The relevant nonlinear processes are discussed in a qualitative model. Experimental results and a rigorous theoretical treatment will be presented in the following sections.

The femtosecond laser system is based on an Er:fiber oscillator mode locked at a repetition rate of $40 \mathrm{MHz}$ and two Er:fiber amplifiers to generate (pump branch) and sample (probe branch) multi-terahertz transients, as depicted in Fig. 1(a). A fiber-coupled electro-optic modulator picks every second pulse in the pump branch, therefore reducing the repetition rate to $20 \mathrm{MHz}$. In both arms, we employ separate highly nonlinear fibers to generate two different supercontinua for the pump and probe which both support pulses with durations of order $10 \mathrm{fs}$ in the near infrared. The pump pulses are centered around a frequency of $193 \mathrm{THz}$ (wavelength of $1550 \mathrm{~nm}$ ) and contain a pulse energy of $4 \mathrm{~nJ}$. Focusing them into a $16-\mu$ m-thin gallium selenide (GaSe) crystal under normal incidence results in the emission of mid-infrared radiation via intrapulse difference-frequency generation (DFG). The terms multiterahertz and mid-infrared (MIR, including the longwave part of the near-infrared accessible to present-day

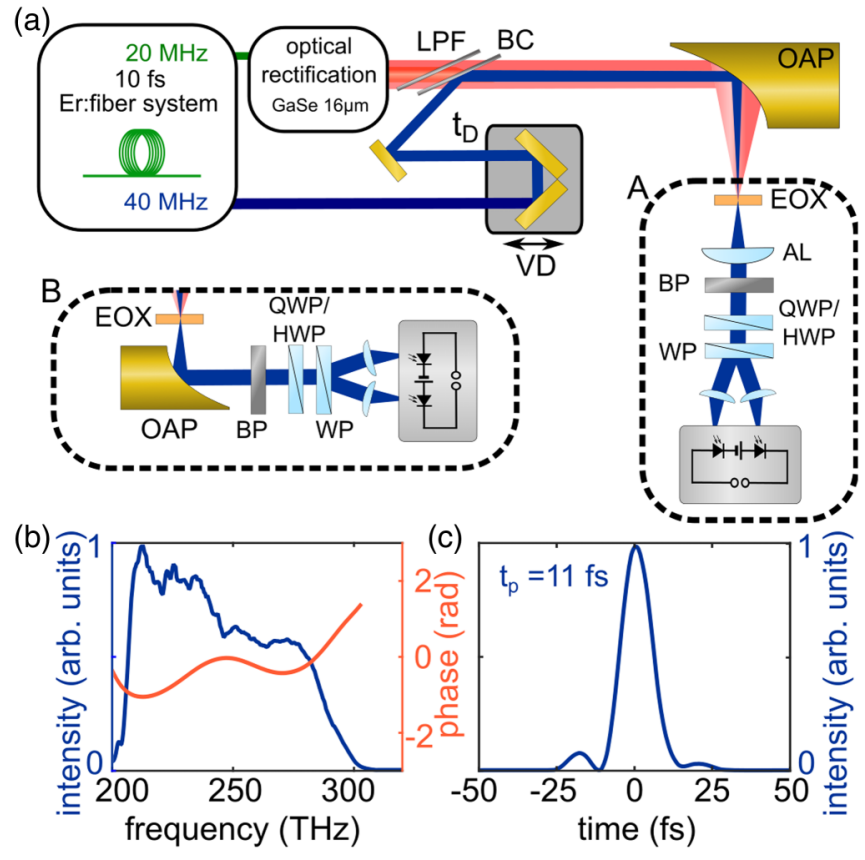

FIG. 1. (a) Scheme of the setup used to generate and detect multiterahertz transients. A femtosecond Er:fiber laser system provides synchronized pump and probe pulse trains at repetition rates of 20 and $40 \mathrm{MHz}$, respectively. The pump branch generates ultrashort mid-infrared transients via optical rectification in a $16-\mu \mathrm{m}$-thick gallium selenide (GaSe) crystal. LPF: optical low-pass filter $(150 \mu \mathrm{m}$ of $\mathrm{GaSb})$. BC: beam combiner $(500 \mu \mathrm{m}$ of $\mathrm{Si})$. OAP: off-axis parabolic mirrors (Au coated). EOX: electro-optic detection crystal (ZnTe, GaSe, or $\mathrm{AgGaS}_{2}$ ). VD: variable optical delay stage to set the timing $t_{D}$ between pump and probe. The probe pulse train is recollimated via an achromatic lens $\mathrm{AL}$ (scheme A) or an OAP (scheme B). Optional bandpass filters (BP), a quarter- or half-wave plate (QWP or HWP), and a Wollaston prim (WP) are followed by a differential photodetector to derive the electro-optic signal $\Delta I / I$. (b) Spectral intensity (dark blue) and phase (light red) of the probe pulses as a function of frequency. (c) Intensity envelope of probe pulses vs time.

electro-optic sampling) will be used synonymously for the remainder of this paper.

A $150 \mu \mathrm{m}$-thick gallium antimonide wafer (LPF) absorbs the remaining near-infrared radiation. Afterwards, a silicon wafer (BC) inserted at the Brewster's angle superimposes probe and multiterahertz beams, and a variable delay (VD) controls the relative timing between both pulse trains. An off-axis parabolic (OAP) mirror focuses these pulse trains to a paraxial spot radius of $3.6 \mu \mathrm{m}$ into an electro-optic detection crystal (EOX) where the mid-infrared electric field couples to the polarization state of the probe photons via the Pockels effect. The probe pulses have a center frequency of $250 \mathrm{THz}$ and contain an energy of $70 \mathrm{pJ}$. The MIR peak field amplitude is on the order of $1 \mathrm{kV} / \mathrm{cm}$. Two different elements may be exploited to recollimate the probe beam: either an achromatic lens (AL, option A marked by dashed black line in Fig. 1(a)] or an OAP (option B). Subsequently, we use a balanced detection scheme consisting of either a quarter(QWP) or a half-wave plate (HWP), a Wollaston prism (WP), and balanced photodiodes to analyze the induced polarization 
changes of the probe beam. A radio-frequency lock-in amplifier enables shot-noise limited recording of the differential photocurrent $\Delta I / I$ by normalizing the signal demodulated at the repetition frequency of the pump of $20 \mathrm{MHz}$ to the total flux of probe photons recorded at $40 \mathrm{MHz}$ [14]. Closely matched bandpass filters (BP) of a bandwidth of $50 \mathrm{~nm}$ may be inserted to resolve the induced polarization changes within the near-infrared probe spectrum. The probe wave packets were characterized by frequency-resolved optical gating [28]. Their spectral amplitude (dark blue) and phase (light red) are depicted in Fig. 1(b). A FWHM pulse duration of $t_{p}=11$ fs of the intensity envelope is demonstrated in Fig. 1(c). As compared to the more broadband probe pulses from, e.g., Refs. [14] and [16], we are working here with a somewhat lower bandwidth and less structured spectra to elucidate the targeted physics in the clearest way possible.

The enabling principle of our experiments is the secondorder nonlinear interaction between the probe and multiterahertz pulses. This process is traditionally interpreted in analogy to the linear electro-optic effect in a Pockels cell. If the probe pulses are much shorter than half an oscillation cycle of the mid-infrared field, the latter may indeed be treated as quasistatic, provided copropagation requirements are satisfied by appropriate phase matching. The nonlinear response of the detection crystal then induces a transient birefringence, causing a phase shift proportional to the strength of the multiterahertz electric field. This process leads to an elliptical polarization of the probe pulse which was linearly polarized at the entrance face of the EOX [29]. Strictly speaking, this description is valid when the multiterahertz frequencies involved are much smaller than the bandwidth of the probe spectrum. For higher multiterahertz frequencies, a more quantitative approach in the frequency domain becomes appropriate [27]. The nonlinear interaction is then described as a combination of DFG and sum frequency generation (SFG). Figure 2 sketches the nonlinear mixing between the near-infrared (NIR) and MIR pulse trains. The incident probe field $\mathbf{E}_{p}$ and multiterahertz field $\mathbf{E}_{\mathrm{THz}}$ are polarized linearly but perpendicular to each other along unit vectors $\boldsymbol{e}_{z}$ and $\boldsymbol{e}_{s}$, respectively. In the geometry typically used for EOS, an orthogonally polarized MIR field along $\boldsymbol{e}_{s}$ interacts via the second-order nonlinearity creating components $\mathbf{E}^{(2)}$ in the spectral range of the probe but with perpendicular polarization.

The electro-optic signal then arises from interference between the original probe photons and the newly generated ones. Therefore, it is necessary to combine both polarization components of the NIR field via projection to new axes. For this purpose, we use a combination of a Wollaston prism and a phase retarder, i.e., a half- or a quarter-wave plate (HWP or QWP). The angle between their optical axis and $\boldsymbol{e}_{z}$ is set to $22.5^{\circ}$ and $45^{\circ}$, respectively, in order to balance the intensities in both output ports of the Wollaston prism. Therefore, the average electro-optic signal measured by the balanced photodetector vanishes if no copropagating multiterahertz field is present in the detection crystal.

The resulting polarization state of the new NIR field $\mathbf{E}_{p}+$ $\mathbf{E}^{(2)}$ depends not only on the absolute values of $\mathbf{E}_{p}$ and $\mathbf{E}^{(2)}$ but also on their relative phase $\varphi$. If $\varphi=\pi / 2$ or $\varphi=$ 0 , the transmitted probe is polarized elliptically [Fig. 2(b)] or linearly [Fig. 2(c)], respectively. Any phase difference (a)

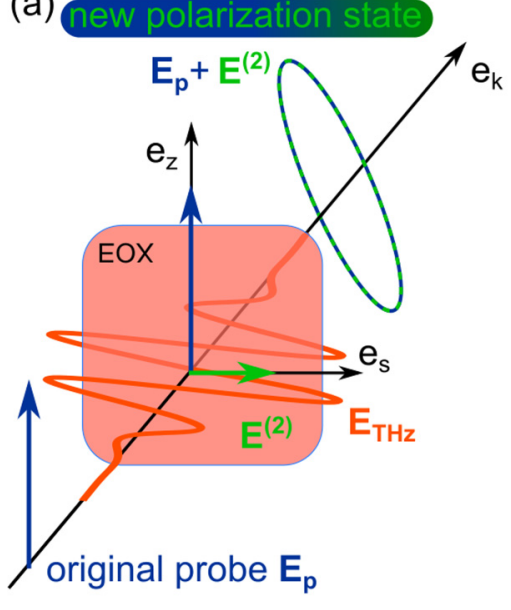

(b)

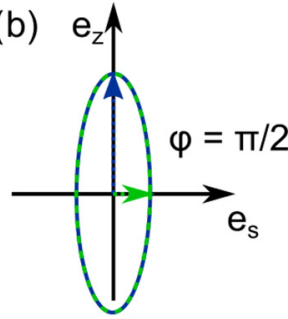

(c)

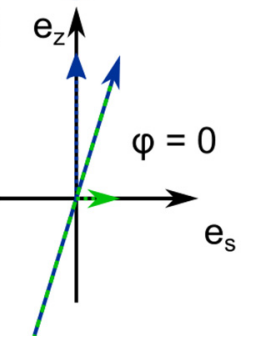

FIG. 2. (a) Illustration of the polarization states of the probe $\mathbf{E}_{p}$ (dark blue arrows), multiterahertz $\mathbf{E}_{\mathrm{THz}}$ (light orange wave form), and newly generated NIR field $\mathbf{E}^{(2)}=\mathbf{E}_{\mathrm{SFG}}+\mathbf{E}_{\mathrm{DFG}}$ (light green). The incident probe and MIR transients are polarized linearly along $\boldsymbol{e}_{z}$ and $\boldsymbol{e}_{s}$, respectively. After nonlinear mixing in the EOX, the resulting NIR field consists of the original probe and SFG/DFG fields which are orthogonally polarized. (b) If $\mathbf{E}_{p}$ and $\mathbf{E}^{(2)}$ are out of phase by an angle $\varphi=\pi / 2$, the total polarization becomes elliptic. The long half axis of the polarization ellipse (dashed blue-green) remains parallel to the stronger component. (c) If the two components are in phase, addition of $\mathbf{E}_{p}$ and $\mathbf{E}^{(2)}$ results in a rotation of the linear polarization (blue-green vector).

$0<\varphi<\pi / 2$ will result in elliptically polarized light with rotated main axes. Since a QWP introduces ellipticity while a HWP rotates the plane of linear polarization, using these two phase retarders in a balanced detection setup [see Fig. 1(a)] measures changes in ellipticity or polarization rotation of the probe, respectively (see Sec. IV). Therefore, in the following we will use the terms "QWP signal" and "HWP signal" for denoting changes in ellipticity and polarization rotation, respectively.

After explaining how the generation of new polarization components via SFG and DFG modifies the probe polarization, we turn our attention to the nonlinear mixing process itself. For this purpose, we will consider only the spectral amplitudes in the polarization directions defined in Fig. 2(a). This step allows us to simplify the treatment of the vector properties of the incoming electric fields. Expressions (1) describe how a new field is generated in an SFG or DFG process between a quasimonochromatic multiterahertz field $E_{\mathrm{THz}}$ and near-infrared probe $E_{p}$ (see Sec. IV for derivation):

$$
\begin{aligned}
& E_{\mathrm{SFG}}\left(\omega, t_{D}\right) \propto i \chi^{(2)} E_{\mathrm{THz}}(\Omega) E_{p}(\omega-\Omega) e^{i\left(-\Omega t_{D}+\varphi_{0}\right)} \\
& E_{\mathrm{DFG}}\left(\omega, t_{D}\right) \propto i \chi^{(2)} E_{\mathrm{THz}}^{*}(\Omega) E_{p}(\omega+\Omega) e^{i\left(\Omega t_{D}+\varphi_{0}\right)},
\end{aligned}
$$

with $\omega$ and $\Omega$ representing the angular frequencies in the near- and mid-infrared spectral regions and $t_{D}$ the relative time delay between probe and multiterahertz pulses. The relevant second-order nonlinear tensor element $\chi^{(2)}$ is considered to be dispersionless in the frequency range of interest, while $E_{\mathrm{THz}}(\Omega)$ and $E_{p}(\omega \pm \Omega)$ are the spectral amplitudes of the multiterahertz and probe fields, respectively. $\varphi_{0}$ tracks any 
additional phase shifts between $E_{\mathrm{SFG} / \mathrm{DFG}}$ and $E_{p}$ occurring, e.g., due to birefringence in the EOX or any other polarizationdependent component in the ellipsometer. Since $E_{\mathrm{SFG} / \mathrm{DFG}}$ and $E_{p}$ are polarized perpendicular to each other, the relevant parameter deciding whether a process in Eq. (1) eventually contributes to the imbalance $\Delta I$ of the photocurrent detected in the differential receiver after the ellipsometer is the phase relative to the broadband probe field polarized along $\boldsymbol{e}_{z}$, i.e., between $E_{\mathrm{SFG}}(\omega)$ and $E_{p}(\omega)$ or $E_{\mathrm{DFG}}(\omega)$ and $E_{p}(\omega)$, respectively. All complex-valued factors in Eq. (1) potentially introduce such a phase shift. In the ideal scenario discussed here assuming bandwidth-limited pulses, real-valued spectral amplitudes $E_{p}(\omega)$ and $E_{\mathrm{THz}}(\Omega)$, a real-valued $\chi^{(2)}$, and negligible phase mismatch, a relative phase of $\pi / 2$ follows from multiplication with the imaginary unit $i$. This fact would result in a purely elliptical deviation from the linear input polarization if $\varphi_{0}=0$. However, during the measurement of a transient via EOS the delay between probing and MIR fields is varied, leading to an additional phase $\pm \Omega t_{D}$. Therefore, the total phase shift for SFG and DFG is

$$
\Delta \varphi_{ \pm}=\frac{\pi}{2}+\varphi_{0} \mp \Omega t_{D} .
$$

At this point, it is important to stress the distinction between delay-dependent and -independent phases in EOS, since a change in $\varphi_{0}$ fundamentally alters the relationships between the QWP and HWP signals.

It is now instructive to visualize the qualitative consequences of Eqs. (1) and (2) for the change of the electrooptic signal $\Delta I / I$ as a function of $t_{D}$ based on a phasor representation in the complex plane (see Fig. 3). The newly generated fields $E_{\mathrm{SFG}}$ and $E_{\mathrm{DFG}}$ are depicted as green arrows of identical length on the left-hand side of Fig. 3. For $t_{D}=0$, $\Delta \varphi_{ \pm}=\pi / 2+\varphi_{0}$, and according to Eq. (1), the phasors for both $E_{\mathrm{SFG}}$ and $E_{\mathrm{DFG}}$ coincide. In the left column of Fig. 3, the real axis, corresponding to the phase of the original probe field $E_{p}$, is taken as the abscissa and the imaginary axis as the ordinate. The projections of the field vector of $E_{\mathrm{DFG}}$ onto the real and imaginary axis then correspond to components which are in phase or out of phase by $\pi / 2$ with the original probe fields [see red and blue dotted lines in the left-hand side of Fig. 3(a), respectively]. They therefore result in a rotation of linear polarization or in an induced ellipticity of the new NIR field $\mathbf{E}_{p}+\mathbf{E}^{(2)}$ after the nonlinear interaction (see Fig. 2). Consequently, the projections of the phasors $E_{\mathrm{SFG}}$ and $E_{\mathrm{DFG}}$ are proportional to their contributions to electrooptic signals when a HWP and a QWP is used as phase retarder, respectively. In Fig. 3(a), we first examine a case where only DFG is relevant, e.g., due to phase matching, and $\varphi_{0}=0$. The phasor then points along the imaginary axis at $t_{D}=0$ and rotates counterclockwise with $t_{D}$. Therefore, we obtain QWP and HWP signals of equal amplitude which are phase shifted by $\pi / 2$ [see blue- and red-dotted graphs in the right-hand side of Fig. 3(a)]. If we now include also SFG [see Fig. 3(b)], due to the opposite sign in Eq. (2) the phasor for $E_{\mathrm{SFG}}$ rotates clockwise with increasing delay time $t_{D}$. Since we assume phase mismatch to be negligible, both mixing processes are equal in amplitude. Therefore, the real part of the combined field amplitude vanishes, eliminating the signal measured in a setup using the HWP [red-dotted graph
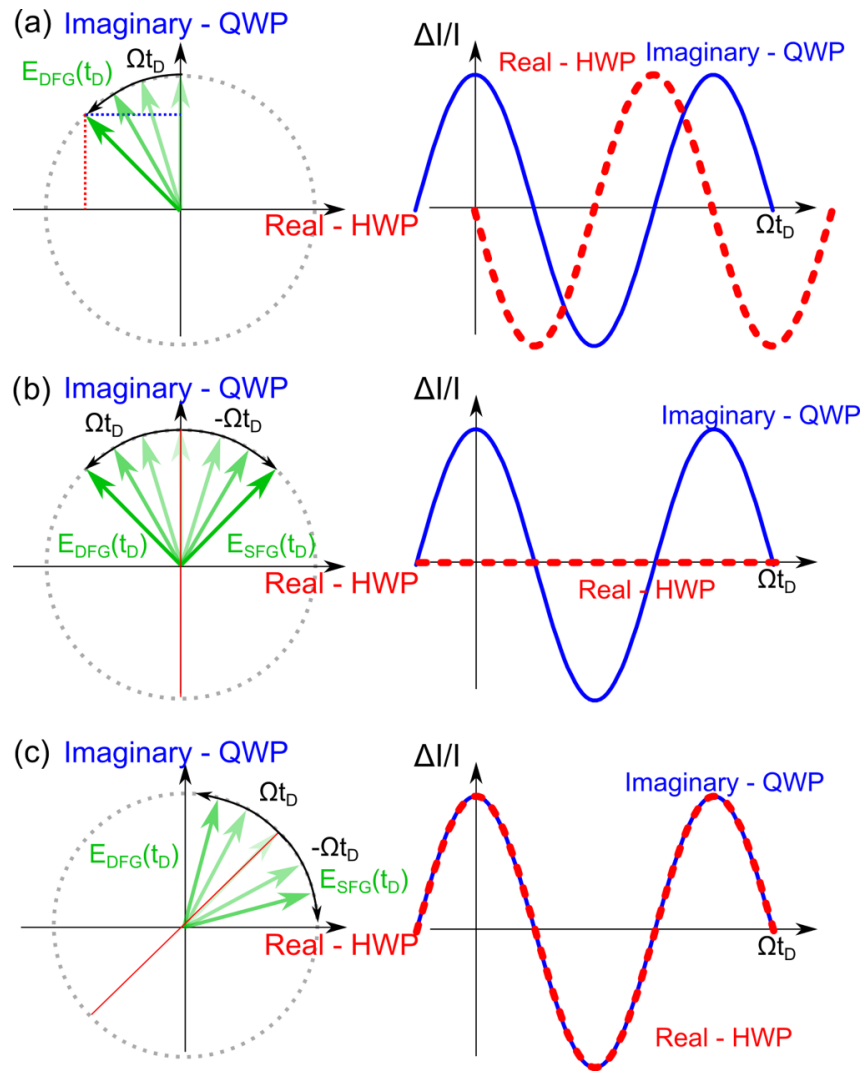

FIG. 3. Left-hand side: phasors (green arrows) of fields in the complex plane generated by sum $\left(E_{\mathrm{SFG}}\right)$ and difference $\left(E_{\mathrm{DFG}}\right)$ frequency generation during EOS. Right-hand side: real and imaginary parts of the combined field $E^{(2)}=E_{\mathrm{SFG}}+E_{\mathrm{DFG}}$, which give rise to electro-optic signals measured using a HWP (red dashed) or a QWP (solid blue line) as a function of delay time between multiterahertz and probe pulses, respectively. (a) Only DFG contributes. QWP and HWP signals are phase shifted by $\pi / 2$ and equal in amplitude. (b) SFG and DFG occur simultaneously. The real part of the combined field cancels for all delays. (c) DFG and SFG contribute, but $\varphi_{0}=$ $-\pi / 4$. Summation of the fields yields identical real and imaginary parts. The red lines in the complex plane indicate the identical directions of the phasors for $E_{\mathrm{SFG}}$ and $E_{\mathrm{DFG}}$ at $t_{D}=0$.

on the right-hand side of Fig. 3(b)]. This scenario recovers the behavior expected from the description of the sampling process based on the static Pockels effect. Note, however, that this finding is no longer valid if $\varphi_{0} \neq 0$. As an example, Fig. 3(c) illustrates the response for an offset phase of $\varphi_{0}=$ $-\pi / 4$. In this case, the projections of the combined field amplitude $E_{\mathrm{SFG}}+E_{\mathrm{DFG}}$ onto the imaginary and real axis are identical for arbitrary delay times. Therefore, the electro-optic signals as measured using a HWP and QWP are expected to coincide. There are several mechanisms which may induce such a delay-independent phase, as detailed in the following sections.

This picture connects the amplitude at a single MIR frequency $\Omega$ with three modes of the probe spectrum, namely, the frequency $\omega$ at which the polarization change is evaluated and the frequencies $\omega \pm \Omega$ contributing to DFG and SFG, respectively. Of course, a full representation of experiments in the time domain involving ultrashort pulses necessitates 


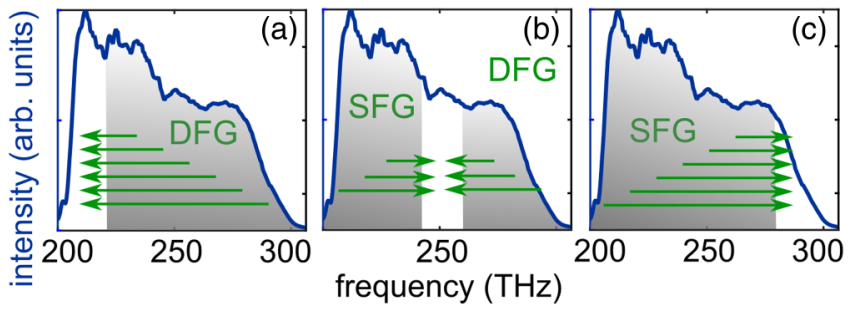

FIG. 4. Spectral intensity of the probe pulses (blue line) filtered after nonlinear interaction with a multiterahertz transient in an EOX. Polarization changes are only analyzed for the white area, while bandpass filters block the probe frequencies in the gray shaded region. Green arrows pointing to the right and left indicate nonlinear frequency conversion via SFG and DFG contributing to the filtered signal, respectively. The starting point of an arrow marks the contributing probe frequency, while its length determines the detected multiterahertz frequency. (a) Filtering low-frequency components, mostly DFG can contribute to the electro-optic signal. (b) When filtering close to the central frequency of the probe spectrum, SFG and DFG contribute symmetrically. (c) Filtering on the high-frequency side favors SFG processes.

integration over all relevant frequencies. Nevertheless, it turns out that our single-mode picture accurately predicts which signal components may be expected in realistic EOS experiments.

Additionally, our approach is valuable to understand the effects of spectral filtering of the probe after the nonlinear interaction with the multiterahertz field. For this purpose, it is essential to recognize that the contributions from SFG and DFG to the electro-optic signal are strongly dependent on the spectral position of polarization analysis of the transmitted probe. This aspect is illustrated in Fig. 4, where the nonlinear mixing within the probe is sketched for three spectral positions selected by different bandpass filters. Gray shaded areas are blocked, while the white areas are transmitted to the ellipsometry setup. The green arrows depict the nonlinear conversion processes that connect frequencies inside the filter bandwidth with the rest of the probe spectrum. The length of the arrows encodes MIR frequencies $\Omega$. Since only DFG processes shift probe photons towards the lower end of the spectrum [see Fig. 4(a)], the contribution of SFG to the electro-optic signal can be largely neglected in this region. The conditions are reversed at the high-frequency side of the probe spectrum [see Fig. 4(c)]. Therefore, signals in the wings of the probe spectrum may be discussed based on the situation sketched in Fig. 3(a), which results in phase-shifted signals of equal amplitude for QWP and HWP. Detection at the center of the probe spectrum largely preserves the symmetry between SFG and DFG [see Fig. 4(b)]. This situation leads to a cancellation of the HWP signal, as visualized in Fig. 3(b). Note that the previous discussion is especially important when the involved multiterahertz frequencies are in the same order of magnitude as the probe bandwidth, i.e., when operating at the highest possible frequency limit for a given sampling pulse. In this case, the spectral position for which SFG and DFG occur symmetrically is restricted to the central part of the probe spectrum.

\section{EXPERIMENTAL RESULTS AND ANALYSIS}

In the previous section, we have developed an intuitive model to describe the method of electro-optic sampling. It allows us to predict polarization rotation and ellipticity changes of the probe field induced by the nonlinear interaction with the multiterahertz transient. We will now proceed with a series of experimental situations selected to elucidate the physics of proper measurements of the electric field and its Hilbert transform. One of the main results of Sec. II is the strong dependence of the expected signals on the balance between SFG and DFG contributions. Therefore, we separate typical electro-optic sensors into two classes: the first group allows favoring one of the processes by exploiting uniaxial birefringence to keep the phase mismatch minimal. Material examples for implementing this scheme are gallium selenide (GaSe), silver gallium sulfide $\left(\mathrm{AgGaS}_{2}\right)$, or $\beta$-barium borate (BBO) $[5,14,7]$. In contrast, crystals isotropic in their linear refractive index such as $\mathrm{ZnTe}$ or GaP may be used as sufficiently thin specimens only $[3,30,4]$. As we shall see, SFG and DFG processes are balanced in this case and are equally relevant for electro-optic detection.

\section{A. Angle phase matching in uniaxial crystals $-\mathbf{A g G a S}_{\mathbf{2}}$}

First, we use a $30-\mu$ m-thick $\mathrm{AgGaS}_{2}$ crystal as a uniaxial electro-optic detector that is critically phase-matched for efficient DFG. The sample is cut at angles of $\theta=53^{\circ}$ and $\varphi=45^{\circ}$. We therefore expect the measurement to perform similar to the scenario depicted in Fig. 3(a). Multiterahertz and probe pulses impinge under normal incidence. Subsequently, an achromatic lens collimates the probe beam [see option A in Fig. 1(a)].

Figure 5(a) depicts electro-optic signals corresponding to ellipticity changes (using a quarter-wave plate, QWP, blue) and polarization rotation (half-wave plate, HWP, red). The measurements show wave forms of comparable amplitude which are phase shifted by approximately $\pi / 2$. This surprising finding contradicts expectations from a simple interpretation based on the static Pockels effect. To investigate further, we spectrally resolve both signals in Figs. 5(b) and 5(c). As discussed in the previous section, spectral filtering leads to differing contributions by SFG and DFG. Generally, if the spectral content of the probe is analyzed above or below its center frequency $(250 \mathrm{THz}$ in our case), the electro-optic signal should be dominated by SFG or DFG, respectively. In our experiment, the low-frequency components dominate the electro-optic signals integrated over the entire bandwidth of the probe, as predicted by phase-matching calculations. Therefore, the phase shift of approximately $\pi / 2$ between QWP and HWP signals occurs in both the spectrally resolved and integrated measurements.

As seen in the inset of Fig. 5(a), the amplitude spectra obtained with both wave-plate configurations are virtually identical, indicating they are Hilbert transforms of each other. This result is fully consistent with our intuitive picture discussed in the previous section, considering only the DFG process [see Fig. 3(a)]. A rigorous analytical and numerical treatment follows in Sec. IV. 


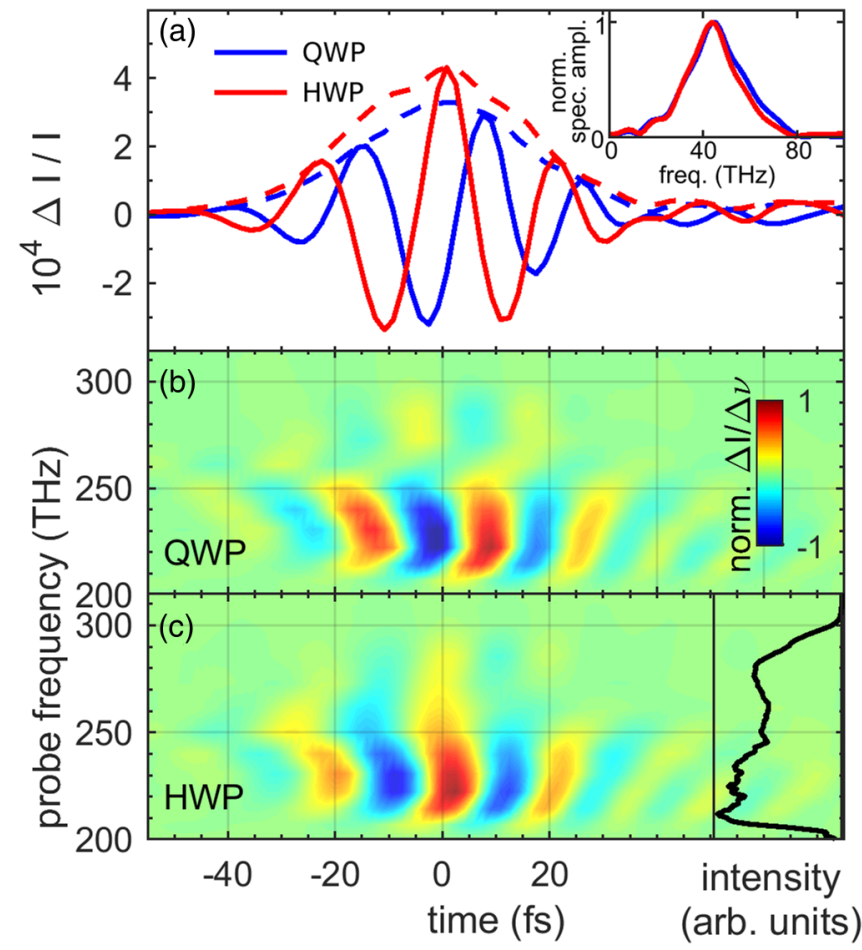

FIG. 5. (a) Electro-optic signals as a function of delay time measured using a $30-\mu$ m-thick $\mathrm{AgGaS}_{2}$ crystal and a quarter- (dark blue) or half-wave plate (light red) in the ellipsometer. The dashed lines show the envelopes of the wave packets. Normalized amplitude spectra are displayed in the inset as a function of multiterahertz frequency. (b), (c) Color-coded electro-optic traces of the spectrally resolved differential photocurrents over the entire probe spectrum as a function of delay time, analyzed using a QWP or HWP, respectively. The signals are normalized to the varying spectral filter bandwidth $\Delta v$. The black graph at the right side of (c) depicts the intensity spectrum of the probe pulses.

The slight difference in peak amplitude between QWP and HWP signals of Fig. 5(a) results from the weak electrooptic signals visible on the high-frequency side of the probe spectrum. These SFG contributions arise due to the fact that the crystal is still relatively thin. Note that there is a phase shift around the center frequency of the probe for the signals acquired using a QWP. Based on Sec. II, one would expect such a phase shift to occur only in HWP measurements which are sensitive to polarization rotation but not to ellipticity changes. The explanation is provided by the static birefringence of the $\mathrm{AgGaS}_{2}$ crystal: the initial near-infrared field components and those generated by nonlinear interaction propagate on different polarization axes. The slightly different optical path lengths modify the delay-independent phase by introducing a finite value for $\varphi_{0}$. Such a change is strongly dependent on the thickness and the birefringent dispersion of the crystal. In the specific case of Fig. 5, it leads to HWP signals that are in phase over the full probe spectrum. Consequently, $\varphi_{0}$ has to be close to $\pm \pi / 2$. Indeed, measurements with a slightly thinner $\mathrm{AgGaS}_{2}$ crystal show smaller phase shifts around the center frequency of the probe (not shown).

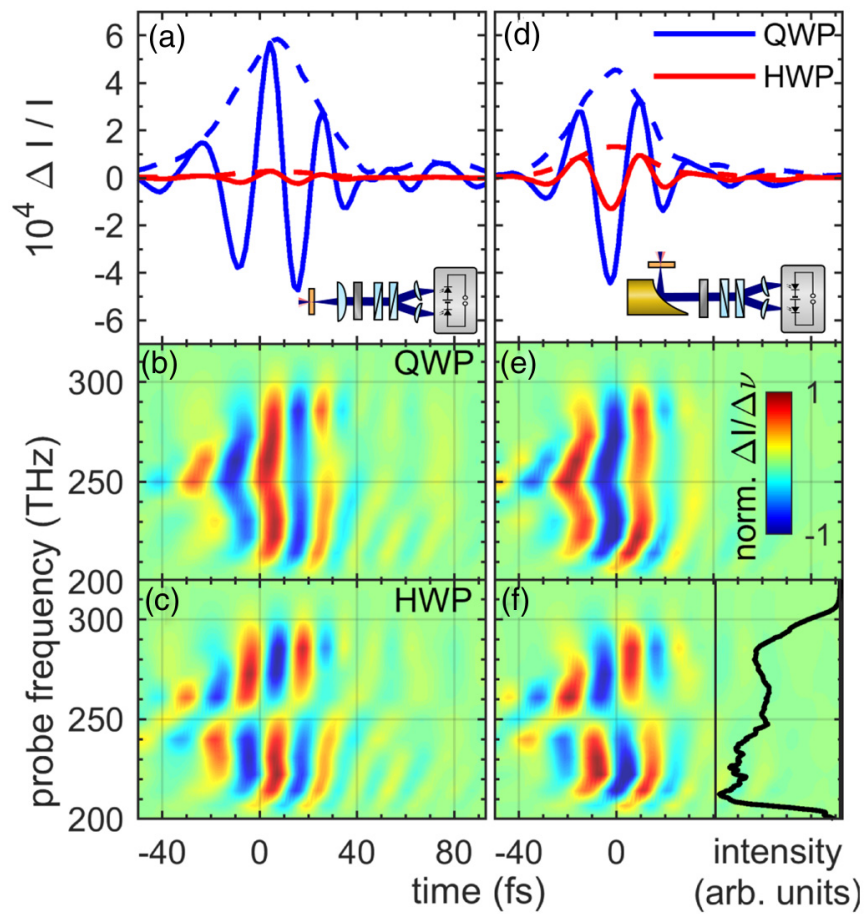

FIG. 6. (a), (d) Electro-optic signals measured using a $15-\mu \mathrm{m}-$ thick ZnTe sample and a quarter- (dark blue, QWP) or half-wave plate (light red, HWP) as a function of delay time while either an achromatic lens or an off-axis parabolic mirror is used for recollimation (see insets for the setup of the ellipsometer). (b), (e) Spectrally resolved difference currents using a QWP corresponding to panels (a) and (d), respectively. (c), (f) Spectrally resolved difference currents using a HWP instead. All spectrally resolved difference currents are normalized to the filter bandwidth $\Delta v$. The probe spectrum is indicated by the black inset in (f).

\section{B. Thin crystals with phase mismatch - ZnTe and GaSe under normal incidence}

We now use ZnTe as an example for an isotropic electrooptic detector. The probe and multiterahertz radiation impinge under normal incidence and parallel to the [110] crystallographic axis of the zincblende-type crystal. Thin samples are necessary to minimize the phase mismatch between the interacting fields. Note that the group and phase velocity dispersion is independent of polarization in this isotropic setting. Consequently, there exists a complementary DFG process for each SFG process within the probe spectrum with exactly the same phase mismatch. None of the two processes is favored when analyzing polarization changes in a spectrally integrated manner. In contrast to the birefringent case of $\mathrm{AgGaS}_{2}$, propagation through the material does not modify the delay-independent phase, and we expect $\varphi_{0}=0$ in Eq. (2) and a behavior close to that depicted in Fig. 3(b).

The consequences are examined with a $15-\mu \mathrm{m}$-thick ZnTe crystal. We analyze polarization rotation and ellipticity changes as discussed above. The left column of Fig. 6 depicts results obtained collimating with an achromatic lens [see inset and Fig. 1(a)]. Figure 6(a) shows electro-optic signals as measured with a quarter- (dark blue) and half-wave plate (light red). The induced ellipticity analyzed with the QWP is 
now one order of magnitude stronger than the polarization rotation measured with the HWP. The spectrally resolved QWP transients are color-coded in Fig. 6(b) and in phase over the full probe spectrum. As discussed below, deviations from straight vertical lines originate predominantly from the loss of sensitivity for high-frequency components in the center of the probe spectrum. The HWP signal amplitudes in Fig. 6(c) are similar to the QWP but invert sign around $250 \mathrm{THz}$ because the sampling process induces an exactly opposite polarization rotation when dominated by SFG as compared to DFG. This result explains the weak spectrally integrated signal [red graph in Fig. 6(a)]. Quite remarkably, these results are fully consistent with our intuitive single-mode picture predicting cancellation of the HWP signal for an ideal delayindependent phase of $\pi / 2$ [see Fig. 3(b)]. In the experiment, the suppression of the spectrally integrated HWP signal is limited by the precision of the retardation of the achromatic half-wave plate.

In the $\mathrm{AgGaS}_{2}$, EOX spectral filtering was provided inherently by phase matching. Additional bandpass filters were employed to provide spectral resolution, showing that DFG dominates in this case. In contrast, explicit filtering is mandatory with a ZnTe EOX to isolate the Hilbert transform of the electric field in the HWP signal, because three-wave mixing in an isotropic crystal maintains the balance between SFG and DFG. As suggested by Fig. 6(c), this task may be provided by bandpass filters in the lower or upper half of the probe spectrum.

The delay-independent phase is identified as a crucial aspect for performing quantitative electro-optic characterization of an electric-field wave form. To examine a scenario where a minute change in this quantity already causes unexpectedly strong effects, we use an off-axis parabolic mirror to collimate the pulse train, as sketched in the inset of Fig. 6(d). Due to the finite imaginary component of the refractive index, reflection on a metallic surface introduces a phase shift that is neither zero nor $\pi$ with respect to the incoming wave [31]. This fact leads to surprisingly pronounced changes in the electro-optic signals, since the phase shift depends on the angle of incidence and the polarization, therefore adding to the delay-independent phase between newly generated and original probe light. As a result, the QWP signal decreases while the HWP increases, as seen in the experimental results depicted in Fig. 6(d) and predicted by our qualitative singlemode picture. The two-dimensional patterns in Figs. 6(e) and 6(f) now feature a significant spectral dependence of the two electro-optic signals. As compared to Fig. 6(a), the weaker spectrally integrated QWP signal is explained by the inclined spectral patterns in Fig. 6(e). Also, the precise temporal overlap of the extrema of the rotation signal above and below the center frequency of the probe spectrum is broken in Fig. 6(f), causing an increase of the electro-optic signal after spectral integration [red graph in Fig. 6(d)]. Again, these observations may be understood in terms of our intuitive single-mode picture: when the delay-independent phase is changed, QWP signals induced by both SFG and DFG symmetrically remain in phase with the electric field [see Fig. 3(c)]. However, if one of the processes dominates, the signal is phase shifted by the delay-independent phase (see Sec. III A). The balance between SFG and DFG in isotropic crystals depends on the
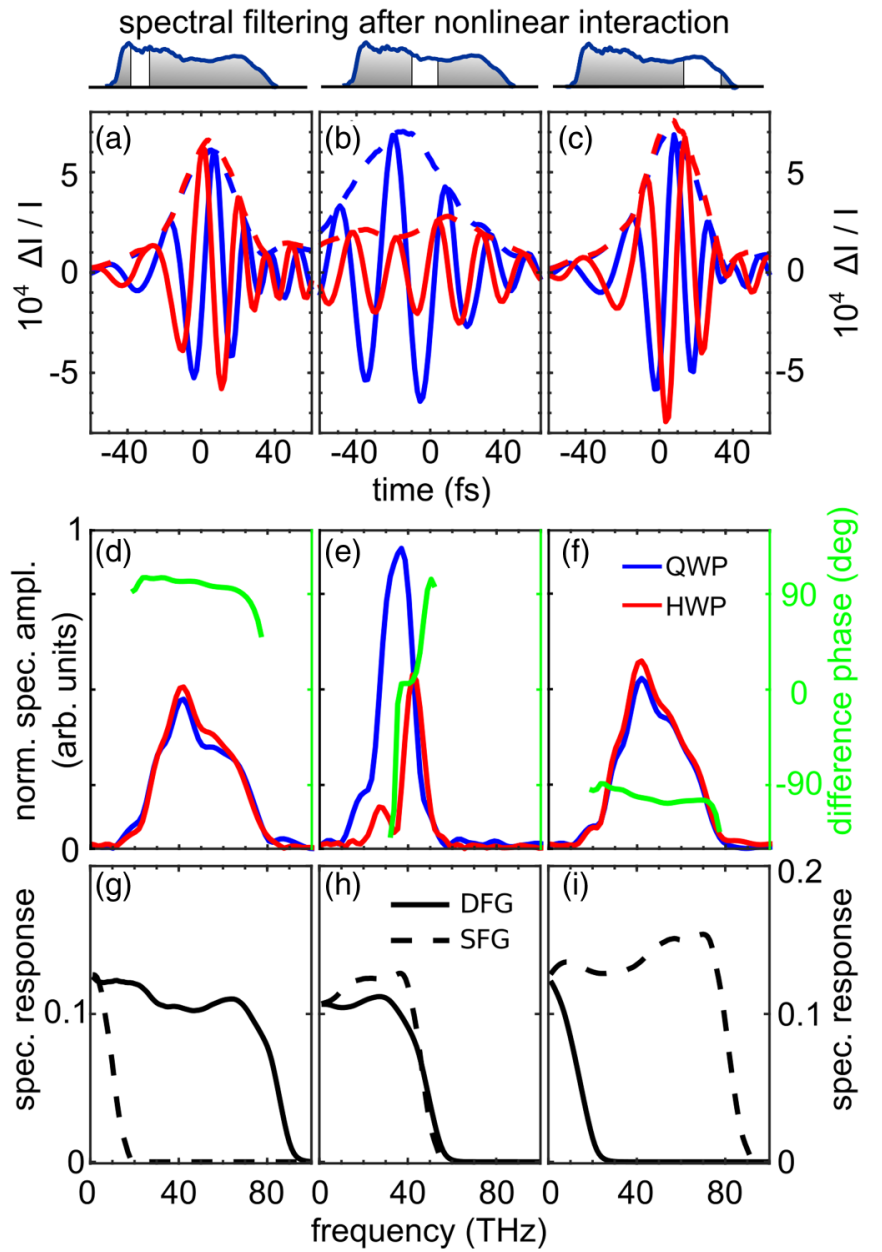

FIG. 7. Spectral filtering of the probe after nonlinear interaction with the multiterahertz field. The sketch in the top row indicates the position of bandpass filters (white areas) within the probe spectrum. Central frequencies are $214 \mathrm{THz}$ (left), $250 \mathrm{THz}$ (center), and 286 $\mathrm{THz}$ (right), respectively. (a)-(c) Electro-optic signals as a function of delay time after application of the bandpass filters indicated above and measured using quarter- (dark blue) and half-wave plates (light red). (d)-(f) Corresponding spectral amplitudes (blue and red) and difference phase between both electro-optic signals (light green). (g)-(i) Normalized spectral response calculated as a function of multiterahertz frequency for contributions of DFG (full lines) and SFG (dashed) to electro-optic signals in the transmission bandwidth of the corresponding filters.

position in the probe spectrum of the frequencies evaluated, introducing a spectral dependence into the frequency-resolved signals. An extensive analytical and numerical discussion on the relationship of the QWP and HWP signals for arbitrary delay-independent phases with the actual multiterahertz may be found in Sec. IV. Also note that other effects such as imperfect input polarization or waveplates must lead to similar effects, further complicating the measurement of the electric field in the time domain. A detailed discussion of this fact may be found in the Appendix.

The measurements under collimation by a lens [sketch in Fig. 6(a)] and therefore $\varphi_{0}=0$ are now examined more closely. Figure 7 depicts multiterahertz transients with three 
different bandpass filters inserted after the EOX, corresponding to horizontal cuts in Figs. 6(b) and 6(c). The sketches in the top row of Fig. 7 show for which probe frequencies the induced polarization changes are sampled. Again, white areas symbolize transmission by the bandpass filters. Red and blue graphs in Figs. 7(a)-7(c) depict the signals due to polarization rotation (HWP) and ellipticity (QWP), respectively. The corresponding amplitude spectra are displayed in Figs. 7(d) and 7(f) with the same color coding. They are virtually identical for HWP and QWP signals. This effect arises because either SFG or DFG dominates the sampling process in the wings of the probe spectrum, as demonstrated in Fig. 4. Additionally, we calculate the difference phase by dividing the Fourier transforms of both transients, which are expected to be

$$
\Delta \phi(\Omega)=\arg \left\{\frac{\mathcal{F}(\mathrm{QWP})}{\mathcal{F}(\mathrm{HWP})}\right\}= \pm \frac{\pi}{2}=\text { const. }
$$

These data are displayed as green graphs in Figs. 7(d) and 7(f). Indeed, relative phase shifts by $\pm \pi / 2$ are found, again indicating that these electro-optic signals are Hilbert transforms of each other. We formally prove this behavior compatible with the standard theory on EOS in Sec. IV. In contrast to the results of Sec. III A, the imbalance here is not a consequence of the phase matching in the EOX but instead of the spectral filtering of the probe after the three-wave mixing.

The amplitude spectra measured close to the center frequency of the probe spectrum [Fig. 7(e)] exhibit a cutoff around $50 \mathrm{THz}$, corresponding to half of the full bandwidth of the probe. Note that the electro-optic signal emerges from interference between field components $E^{(2)}(\omega)$ shifted to the NIR target frequency $\omega$ and the original probe field $E_{p}(\omega)$ which did not interact with the multiterahertz radiation [see Fig. 4]. A photon in the multiterahertz range at frequency $\Omega$ may be detected if it bridges the gap between two probe components with finite amplitude at frequencies $\omega$ and $\omega$ $\pm \Omega$. Filtering after the interaction restricts $\omega$ to, e.g., the center of the probe spectrum and therefore the highest multiterahertz frequency $\Omega$ detectable corresponds to half the probe bandwidth [see Fig. 7(e)]. Another consequence of filtering in the center is that we measure only a very weak polarization rotation for multiterahertz frequencies below $40 \mathrm{THz}$ [red graph in Fig. 7(e)]. For these low frequencies, SFG and DFG processes are mostly symmetric, eventually leading to only a small spectral amplitude also of the HWP signal in Fig. 7(b). The increase of the spectral amplitude at around $45 \mathrm{THz}$ is explained by the slight displacement of the filter from the actual center of the probe spectrum. For filtering on the highor low-frequency side of the spectrum, the difference between the transmitted frequencies and the minimum or maximum probe frequency dictates the maximum detected frequency. Figure 4 illustrates these facts schematically. For this reason, the cutoff at around $50 \mathrm{THz}$ is absent in Figs. 7(d) and 7(f) but the spectra extend up to $80 \mathrm{THz}$, which is close to the full bandwidth of the probe.

In addition to $\mathrm{ZnTe}$, we exploit an extremely thin piece of $\mathrm{GaSe}$ as the electro-optic detector. This material consists of hexagonal atomic bilayers van-der-Waals bonded to each other, resulting in birefringence with an optical axis oriented

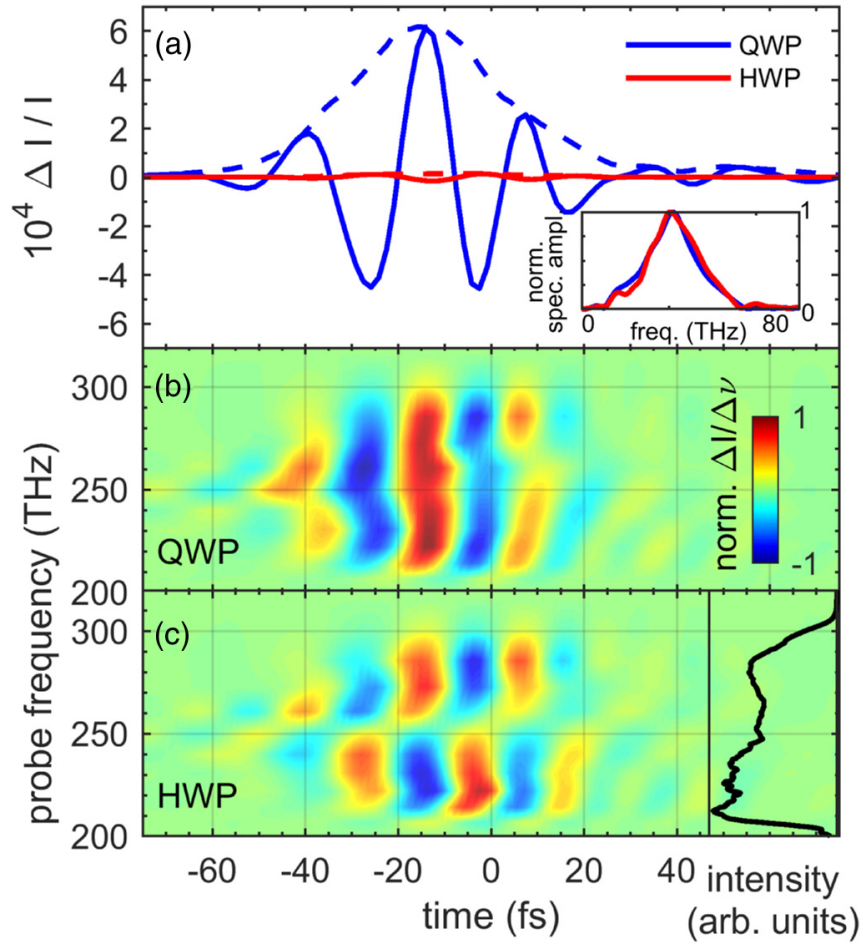

FIG. 8. (a) Electro-optic signals as a function of delay time measured using a 9- $\mu \mathrm{m}$-thick GaSe crystal and a quarter- (dark blue) or half-wave plate (light red) in the ellipsometer. The dashed lines depict the envelopes of the wave packets. Normalized amplitude spectra are depicted as a function of multiterahertz frequency in the inset. (b) Differential photocurrents color-coded vs delay time and probe frequency, as measured with a QWP. The amplitudes are normalized to the filter bandwidth. (c) Same measurement taken with a HWP in the ellipsometer. Black graph at right: spectral intensity of the probe.

perpendicularly to the layering. Therefore, an exfoliated freestanding specimen of $9 \mu \mathrm{m}$ thickness is employed under normal incidence, i.e., with propagation direction along the optical axis. Thus, the refractive index experienced by the beams is isotropic and a finite but identical phase mismatch occurs for SFG and DFG. Figure 8(a) shows multiterahertz transients using QWP and HWP. As in ZnTe, the QWP signal exceeds the HWP signal by one order of magnitude with virtually identical amplitude spectra seen in the inset. Spectrally resolved measurements depicted in Figs. 8(b) (QWP) and 8(c) (HWP) feature comparable amplitudes. The QWP signals are in phase over the entire bandwidth of the probe spectrum [shown as a black line in the right part of Fig 8(c)], while the HWP signals invert sign at around $250 \mathrm{THz}$, again leading to a weak spectral integral. These results are very similar to those obtained with ZnTe in Figs. 6(a)-6(c). Due to the extremely thin EOX, they come even closer to the ideal scenario sketched in Fig. 3(b). This fact underscores the fact that the basic characteristics of the terahertz-induced polarization changes are solely due to the balance between SFG and DFG contributions and are independent of the symmetry of the nonlinear tensor as long as isotropic propagation conditions are ensured. 


\section{RIGOROUS ANALYSIS OF ULTRABROADBAND ELECTRO-OPTIC SAMPLING AND NUMERICAL EVALUATION}

In this section, we quantitatively analyze how SFG and DFG processes result in the polarization changes leading to the electro-optic signals.

\section{A. Balanced detection with QWP and HWP}

First, the electric fields generated via SFG and DFG are projected to the fundamental axes of the Wollaston prism $\boldsymbol{e}_{z}$ and $\boldsymbol{e}_{s}$. The Jones formalism is exploited [32] to express the polarization states by the field amplitudes defined in Fig. 2 and discussed in Sec. II:

$$
\mathbf{E}_{\mathrm{NIR}}=\left(\begin{array}{c}
E^{(2)} \\
E_{p}
\end{array}\right)=\left(\begin{array}{c}
E_{\mathrm{SFG}}+E_{\mathrm{DFG}} \\
E_{p}
\end{array}\right) .
$$

$\mathbf{E}_{\mathrm{NIR}}$ denotes the Jones vector in the $\boldsymbol{e}_{z}$ and $\boldsymbol{e}_{s}$ basis corresponding to the combined near-infrared electric field after the nonlinear interaction in the electro-optic sensor. Now, we consider the action of an arbitrary retarder, introducing a phase shift of $P$ with its fast axis rotated by an angle of $\alpha$ against $\boldsymbol{e}_{z}$ :

$$
\mathbf{E}_{\mathrm{WP}}=\left(\begin{array}{l}
E_{\mathrm{WP}}^{s} \\
E_{\mathrm{WP}}^{z}
\end{array}\right)=M(-\alpha)\left(\begin{array}{cc}
e^{\left(-i \frac{P}{2}\right)} & 0 \\
0 & e^{\left(i \frac{P}{2}\right)}
\end{array}\right) M(\alpha)\left(\begin{array}{c}
E^{(2)} \\
E_{p}
\end{array}\right),
$$

with the rotation matrix

$$
M(\alpha)=\left(\begin{array}{cc}
\cos \alpha & -\sin \alpha \\
\sin \alpha & \cos \alpha
\end{array}\right) .
$$

$\mathbf{E}_{\mathrm{WP}}$ is the complex field amplitude at the Wollaston prism, which separates the two linear polarization components along $\boldsymbol{e}_{z}$ and $\boldsymbol{e}_{s}$. Evaluation of Eq. (5) allows calculation of the optical intensities on the photodiodes for arbitrary retardation and rotation of the fast axis. The difference between the two photocurrents measured on the diodes $\Delta I=I_{z}-I_{s} \propto$ $\left|E_{\mathrm{WP}}^{z}\right|^{2}-\left|E_{\mathrm{WP}}^{s}\right|^{2}$ now results as

$$
\begin{aligned}
\Delta I \propto & \left(\left|E_{p}\right|^{2}-\left|E^{(2)}\right|^{2}\right) \cos ^{2}\left(\frac{P}{2}\right) \\
& +\left(\left|E_{p}\right|^{2}-\left|E^{(2)}\right|^{2}\right) \sin ^{2}\left(\frac{P}{2}\right) \cos (4 \alpha) \\
& +2 \operatorname{Re}\left(E_{p}^{*} E^{(2)}\right) \sin ^{2}\left(\frac{P}{2}\right) \sin (4 \alpha) \\
& +2 \operatorname{Im}\left(E_{p}^{*} E^{(2)}\right) \sin (P) \sin (2 \alpha) .
\end{aligned}
$$

Note that similar expressions have been derived in the context of rotating compensator ellipsometry [33,34]. In the standard configuration with a QWP, $P=\pi / 2$ and $\alpha=\pi / 4$. When calculating the electro-optic signal with Eq. (6), all terms except the last vanish or cancel:

$$
\Delta I_{\mathrm{QWP}}\left(\omega, t_{D}\right) \propto 2 \operatorname{Im}\left[E_{p}^{*}(\omega) E^{(2)}\left(\omega, t_{D}\right)\right] .
$$

For measurements with the HWP, $P=\pi, \alpha=\pi / 8$, and only the third term in Eq. (6) remains finite:

$$
\Delta I_{\mathrm{HWP}}\left(\omega, t_{D}\right) \propto 2 \operatorname{Re}\left[E_{p}^{*}(\omega) E^{(2)}\left(\omega, t_{D}\right)\right] .
$$

Consequently, differential photocurrents measured with a QWP or a HWP are phase shifted by $\pi / 2$ and therefore are Hilbert transforms of each other.

Here we assume a linearly polarized multiterahertz field. The case of arbitrary input polarization is discussed in Ref. [35]. Since $E^{(2)}=E_{\mathrm{SFG}}+E_{\mathrm{DFG}}$, we now investigate how those components emerge from the nonlinear mixing [27]:

$$
\begin{aligned}
E_{\mathrm{SFG}}\left(\omega, t_{D}\right)= & i \int_{0}^{\infty} \frac{2 \pi \omega^{2}}{c^{2} k(\omega)} \chi^{(2)}(\omega ; \Omega ; \omega-\Omega) \zeta_{+} \\
& \times E_{p}(\omega-\Omega) E_{\mathrm{THz}}(\Omega) e^{i\left(-\Omega t_{D}+\varphi_{0}\right)} d \Omega \\
E_{\mathrm{DFG}}\left(\omega, t_{D}\right)= & i \int_{0}^{\infty} \frac{2 \pi \omega^{2}}{c^{2} k(\omega)} \chi^{(2)}(\omega ;-\Omega ; \omega+\Omega) \zeta_{-} \\
& \times E_{p}(\omega+\Omega) E_{\mathrm{THz}}^{*}(\Omega) e^{i\left(\Omega t_{D}+\varphi_{0}\right)} d \Omega .
\end{aligned}
$$

Again, $\omega$ and $\Omega$ represent the angular frequencies in the NIR (probe) and MIR (multiterahertz input) spectral regions, respectively. Note that all spectral amplitudes are defined for positive frequencies only. $t_{D}$ is the relative time delay between the probe and multiterahertz pulses and $\varphi_{0}$ an additional phase that may be added even after the nonlinear mixing. Consistent with Sec. II, the total delay-independent phase is, therefore, $\pi / 2+\varphi_{0}$. The relevant nonlinear tensor element is $\chi^{(2)}$, while $E_{\mathrm{THz}}(\Omega)$ and $E_{p}(\omega \pm \Omega)$ are the spectral amplitudes of the multiterahertz and probe fields, respectively. The phasematching factors $\zeta_{+}$for SFG and $\zeta_{-}$for DFG are defined as

$$
\zeta_{ \pm}=\frac{\exp \left[i \Delta k_{ \pm}(\omega, \Omega) l\right]-1}{i \Delta k_{ \pm}(\omega, \Omega)},
$$

where $l$ is the thickness of the electro-optic crystal, and $\Delta k_{ \pm}$represents the phase mismatch in sum and difference-frequency generation, respectively:

$$
\begin{aligned}
& \Delta k_{+}(\omega, \Omega)=-k(\omega)+k(\Omega)+k(\omega-\Omega), \\
& \Delta k_{-}(\omega, \Omega)=-k(\omega)-k(\Omega)+k(\omega+\Omega) .
\end{aligned}
$$

All wave vectors and the nonlinear tensor elements $\chi^{(2)}$ are taken as real-valued because the frequencies involved in EOS typically avoid dissipative regions of the sensor materials like interband transitions and reststrahlen bands. A model for the dispersion of the complex-valued nonlinearity may be found in Ref. [4]. In the following, we derive the signals expected for specific experimental settings.

\section{B. Identical contributions from SFG and DFG}

First, we examine the SFG and DFG contributions to Eqs. (7) and (8) to see how the interference signals relate to the input electric field. For the HWP signal we use $\operatorname{Re}(a+b)=$ $\operatorname{Re}\left(a+b^{*}\right)$ and obtain

$$
\begin{aligned}
& \Delta I_{\mathrm{HWP}}\left(\omega, t_{D}\right) \propto \operatorname{Re}\left[E_{p}^{*}(\omega) E_{\mathrm{SFG}}\left(\omega, t_{D}\right)\right. \\
& \left.\quad+E_{p}(\omega) E_{\mathrm{DFG}}^{*}\left(\omega, t_{D}\right)\right] .
\end{aligned}
$$


Integration over the NIR probe frequencies $\omega$ yields the total differential signals. We now exploit Eqs. (9) and (10) to insert the explicit terms for SFG and DFG into Eq. (14) and integrate over all NIR frequencies:

$$
\begin{aligned}
\Delta I_{\mathrm{HWP}}\left(t_{D}\right) & =\int_{0}^{\infty} d \omega \frac{\omega_{c}}{\omega} \Delta I_{\mathrm{HWP}}\left(\omega, t_{D}\right) \\
& \propto \int_{0}^{\infty} d \Omega \operatorname{Re}[\underbrace{\left(i \int_{\Omega}^{\infty} d \omega \zeta_{+} E_{p}^{*}(\omega) E_{p}(\omega-\Omega) e^{i \varphi_{0}}-i \int_{0}^{\infty} d \omega \zeta_{-}^{*} E_{p}(\omega) E_{p}^{*}(\omega+\Omega) e^{-i \varphi_{0}}\right)}_{=: R_{\mathrm{HWP}}(\Omega)} E_{\mathrm{THz}}(\Omega) e^{-i \Omega t_{D}}] .
\end{aligned}
$$

This expression connects the expected differential signal with the actual amplitudes of the NIR and MIR fields. Here, a factor of $\omega_{c} / \omega$ with the central frequency $\omega_{c}$ is introduced, since in the actual experiment the difference in photon numbers rather than intensities is detected. The spectral response function $R_{\mathrm{HWP}}(\Omega)$ represents a useful concept for an intuitive physical understanding. It quantifies the relative sensitivity of the sampling process for each multiterahertz input frequency $\Omega$. $R_{\mathrm{HWP}}$ consists of two terms originating from SFG and $\mathrm{DFG}$, respectively. It may be simplified further by substituting $\bar{\omega}=\omega-\Omega$ in the first integral:

$$
\begin{aligned}
R_{\mathrm{HWP}}(\Omega)= & i \int_{0}^{\infty} d \bar{\omega} \zeta_{+} E_{p}^{*}(\bar{\omega}+\Omega) E_{p}(\bar{\omega}) e^{i \varphi_{0}} \\
& -i \int_{0}^{\infty} d \omega \zeta_{-}^{*} E_{p}(\omega) E_{p}^{*}(\omega+\Omega) e^{-i \varphi_{0}} .
\end{aligned}
$$

In case of an isotropic refractive index, these two integrals may be combined. The phase-matching term transforms as

$$
\zeta\left[\Delta k_{+}(\bar{\omega}+\Omega, \Omega)\right]=\zeta\left(-\Delta k_{-}\right)=\zeta^{*}\left(\Delta k_{-}\right)
$$

because

$$
\Delta k_{+}(\bar{\omega}+\Omega, \Omega)=-\Delta k_{-}(\bar{\omega}, \Omega) .
$$

With this result, we may write

$$
\begin{aligned}
R_{\mathrm{HWP}}(\Omega) & =\left(i e^{i \varphi_{0}}-i e^{-i \varphi_{0}}\right) \int_{0}^{\infty} d \omega \zeta_{-}^{*} E_{p}(\omega) E_{p}^{*}(\omega+\Omega) \\
& =-2 \sin \left(\varphi_{0}\right) R(\Omega)
\end{aligned}
$$

and the generalized response function becomes

$$
R(\Omega)=\int_{0}^{\infty} d \omega \zeta_{-}^{*} E_{p}(\omega) E_{p}^{*}(\omega+\Omega) .
$$

Physically, this result means that when integrating over the full probe spectrum it is possible to describe the sampling process with an expression that looks like it exclusively contains DFG. This curious fact is understandable in materials with an isotropic refractive index. Here, the dispersion relations for the polarizations of the probe field and the generated field are identical. Consequently, for every DFG process there exists an SFG counterpart representing the time-reversed process with the same three frequencies and wave vectors. This SFG component may be expressed as a Hermitian conjugated DFG process. Note that this is only possible if the difference in photon numbers is detected. Comparing the intensities would lead to a bias for higher frequencies, which would favor the SFG contribution. Pairing off the SFG and DFG contributions in this manner, a generalized response function $R(\Omega)$ is defined. It includes a spectral autocorrelation of the probe field modified by the phase-matching factor $\zeta_{-}$.

For the QWP signal $\Delta I_{\mathrm{QWP}}$, a similar derivation consistent with the theory of standard EOS [27] is performed. We exploit Eq. (9) and $\operatorname{Im}(a+b)=\operatorname{Im}\left(a-b^{*}\right)$ to define

$$
\begin{aligned}
R_{\mathrm{QWP}}= & i \int_{\Omega}^{\infty} d \omega \zeta_{+} E_{p}^{*}(\omega) E_{p}(\omega-\Omega) e^{i \varphi_{0}} \\
& +i \int_{0}^{\infty} d \omega \zeta_{-}^{*} E_{p}(\omega) E_{p}^{*}(\omega+\Omega) e^{-i \varphi_{0}} .
\end{aligned}
$$

Substitution analogous to the previous derivation allows to write everything in terms of the general response function:

$$
\begin{aligned}
R_{\mathrm{QWP}}(\Omega) & =i\left(e^{i \varphi_{0}}+e^{\left.-i \varphi_{0}\right)}\right) \int_{0}^{\infty} d \omega \zeta_{-}^{*} E_{p}(\omega) E_{p}^{*}(\omega+\Omega) \\
& =2 i \cos \left(\varphi_{0}\right) R(\Omega)
\end{aligned}
$$

To conclude, QWP and HWP signals are in phase with each other and with the multiterahertz electric field for a sufficiently thin electro-optic detector and bandwidth-limited probe pulses. They are given by

$$
\Delta I_{\mathrm{QWP}}\left(t_{D}\right) \propto 2 \cos \left(\varphi_{0}\right) \int_{0}^{\infty} d \Omega \operatorname{Re}\left[R(\Omega) E_{\mathrm{THz}}(\Omega) e^{-i \Omega t_{D}}\right],
$$

$\Delta I_{\mathrm{HWP}}\left(t_{D}\right) \propto-2 \sin \left(\varphi_{0}\right) \int_{0}^{\infty} d \Omega \operatorname{Re}\left[R(\Omega) E_{\mathrm{THz}}(\Omega) e^{-i \Omega t_{D}}\right]$.

These findings agree with the measured data displayed in Figs. 6(a) and 8(a). In the ideal case, $\varphi_{0}=0$. A deviation from this scenario may arise even after the nonlinear interaction [compare Fig. 6(d) and Sec. III B], leading to a finite polarization rotation, as measured with the HWP. At the same time, the amplitude of the QWP signal decreases. We may write

$$
\frac{\Delta I_{\mathrm{QWP}}}{\Delta I_{\mathrm{HWP}}}=-\frac{\cos \left(\varphi_{0}\right)}{\sin \left(\varphi_{0}\right)}=-\cot \left(\varphi_{0}\right)
$$

Note that phase mismatch in the electro-optic crystal may change the relationship of the QWP and HWP signals with respect to the multiterahertz field but not with each other.

\section{EOS based on DFG only}

In the case of $\Delta k_{+} \gg \Delta k_{.-} \approx 0$ the contribution of the SFG process vanishes and the derivation of electro-optic signals corresponding to polarization rotation and ellipticity becomes straightforward. By inserting exclusively Eq. (10) 
into Eqs. (7) and (8) and taking the real part we obtain

$$
\begin{aligned}
& \Delta I_{\mathrm{QWP}}\left(t_{D}\right) \propto \int_{0}^{\infty} d \Omega \operatorname{Re}\left[R(\Omega) E_{\mathrm{THz}}(\Omega) e^{-i\left(\Omega t_{D}+\varphi_{0}\right)}\right], \\
& \Delta I_{\mathrm{HWP}}\left(t_{D}\right) \propto \int_{0}^{\infty} d \Omega \operatorname{Re}\left[R(\Omega) E_{\mathrm{THz}}(\Omega) e^{-i\left(\Omega t_{D}+\varphi_{0}+\pi / 2\right)}\right] .
\end{aligned}
$$

Here both signals exhibit the same peak amplitude independent of $\varphi_{0}$. However, the QWP signal is exactly in phase with the multiterahertz electric field only for $\varphi_{0}=0$. Any deviation leads to an equivalent phase shift of the measured transient. The same phase shift occurs in the HWP signal, yet it is always shifted by $\pi / 2$ against the QWP signal. This finding supports the conclusions drawn from the intuitive picture [see Fig. 3(a)] and the measurement using $\mathrm{AgGaS}_{2}$ as an EOX [see Fig. 5(a)]: HWP and QWP configurations measure signals with identical amplitude but a phase shift of $\pi / 2$ when only one process contributes to the polarization changes. Note that the choice to express the sampling process via DFG is arbitrary, and a measurement using only the SFG process would change only the sign of the phase shift of the HWP signal. To conclude, these theoretical results are in excellent agreement with the spectrally integrated data presented in Sec. III.

\section{Spectral response after probe filtering}

The generalized response function combining SFG and DFG contributions (in Sec. IV B) is a result of integration over the full probe spectrum. Filtering after the nonlinear interaction breaks the symmetry between both processes and therefore, different normalized response functions arise:

$$
\begin{aligned}
& \tilde{R}_{\mathrm{DFG}}(\Omega)=\frac{1}{N} \int_{\omega_{1}}^{\omega_{2}} d \omega \zeta_{-}^{*} E_{p}(\omega) E_{p}^{*}(\omega+\Omega), \\
& \tilde{R}_{\mathrm{SFG}}(\Omega)=\frac{1}{N} \int_{\omega_{1}}^{\omega_{2}} d \omega \zeta_{+} E_{p}^{*}(\omega) E_{p}(\omega-\Omega),
\end{aligned}
$$

with the cut-off frequencies of the filter $\omega_{1}$ and $\omega_{2} . E_{p}(\omega)$ is the complex electric field amplitude of the probe. The normalization constant $N=\int_{0}^{\infty} d \omega\left|\omega_{c} / \omega E_{p}(\omega)\right|^{2}$ is proportional to the total energy of the probe. This normalization is relevant when computing the absolute differential photocurrent as displayed in the color-coded graphs of this section. In any case, the autocorrelation leads to a continuous roll-off of the response function for typical shapes of the probe spectrum. This fact results in an increased sensitivity for lower frequencies and a corresponding redshift of the amplitude spectra recorded as compared to the input multiterahertz field. However, two modifications occur when spectral filters are applied after the nonlinear interaction: the response functions for SFG and DFG differ and the continuous roll-off is modified towards a step function. Again, Fig. 4 is helpful for an intuitive understanding of this fact: detection of low frequencies $\Omega$ is possible in any spectral region of the probe, while the information about the maximal frequencies covered by the probe bandwidth is contained only in the spectral wings. Restricting the spectral bandwidth contributing to the signal tends to equalize the sensitivities for different input frequencies $\Omega$. The cutoff at high frequencies is given by the position of the spectral filter. To illustrate these findings, we apply our formalism to the data depicted in Figs. 7(a)-7(f) to compute the response functions as given by Eq. (28) using the measured amplitude spectrum of the probe and the transmission bandwidth of the bandpass filters used for these measurements. Figures 7(g)-7(i) depict the result of these calculations. They quantify how sensitive the contributions of SFG (dashed black lines) and DFG (solid black lines) to QWP and HWP signals are as a function of multiterahertz frequency. The intuitive picture developed in Sec. II with its phasor representation shows that in case of $\varphi_{0}=0$, SFG and DFG contributions add up to generate a QWP signal, while they subtract for the HWP signal. Formally, this behavior is encoded in the corresponding signs between the terms from SFG and DFG in the original definitions of the response functions in Eqs. (15) and (21). It follows that the spectral response for both processes must also be added or subtracted for QWP and HWP signals, respectively. Therefore, either DFG or SFG dominates the polarization changes in the low- or high-frequency regions of the probe spectrum [Figs. 7(g) and 7(i)]. By symmetry, the response functions for DFG and SFG become virtually identical in the center of the probe spectrum [Fig. 7(h)]. In our setup, the contributions for frequencies below approximately $15 \mathrm{THz}$, corresponding to wavelengths longer than $20 \mu \mathrm{m}$, are suppressed due to diffraction losses between emitter and detector. We emphasize that these restrictions on the detection bandwidth are universal to all measurements in electro-optic sampling, with or without spectral filtering and regardless of the type of EOX. This finding follows from the fact that they are a direct consequence of the limited bandwidth of the probe. Therefore, when one is interested in high frequencies, it may be valuable to filter the probe spectrum for enhancing the signal-to-noise ratio $[7,36]$. However, assuming $\varphi_{0}=0$, all spectrally resolved measurements are in phase [see Fig. 6(b)]. Consequently, spectrally integrated measurements using a QWP always exhibit the full sensitivity for high multiterahertz frequencies. A relative increase of the signal in this spectral range must be understood as a result of attenuating lower frequency components.

\section{E. Numerical calculations for frequency-resolved EOS - Delay-independent phase}

In this subsection, we numerically evaluate the electrooptic sampling to study the QWP and HWP signals as a function of probe frequency. Specifically we compute Eqs. (7) to (10) to provide an additional check on the results presented above. For this purpose, we assume a Gaussian-shaped multiterahertz spectrum at around $25 \mathrm{THz}$ with a FWHM of $16 \mathrm{THz}$. The probe covers a spectral width of $75 \mathrm{THz}$ centered at $250 \mathrm{THz}$, significantly surpassing the bandwidth required for adequate sampling of the input wave form. In this way, the effects of a strongly varying frequency response are minimized, enabling a straightforward comparison in the time domain. The spectral phases for both fields are taken to be zero. All simulations shown in the following are calculated for identical input spectra, and all results are normalized to the same values for the entire section. In contrast to experimental results presented in the previous sections, here we have the full knowledge about the time evolution of the input electric 

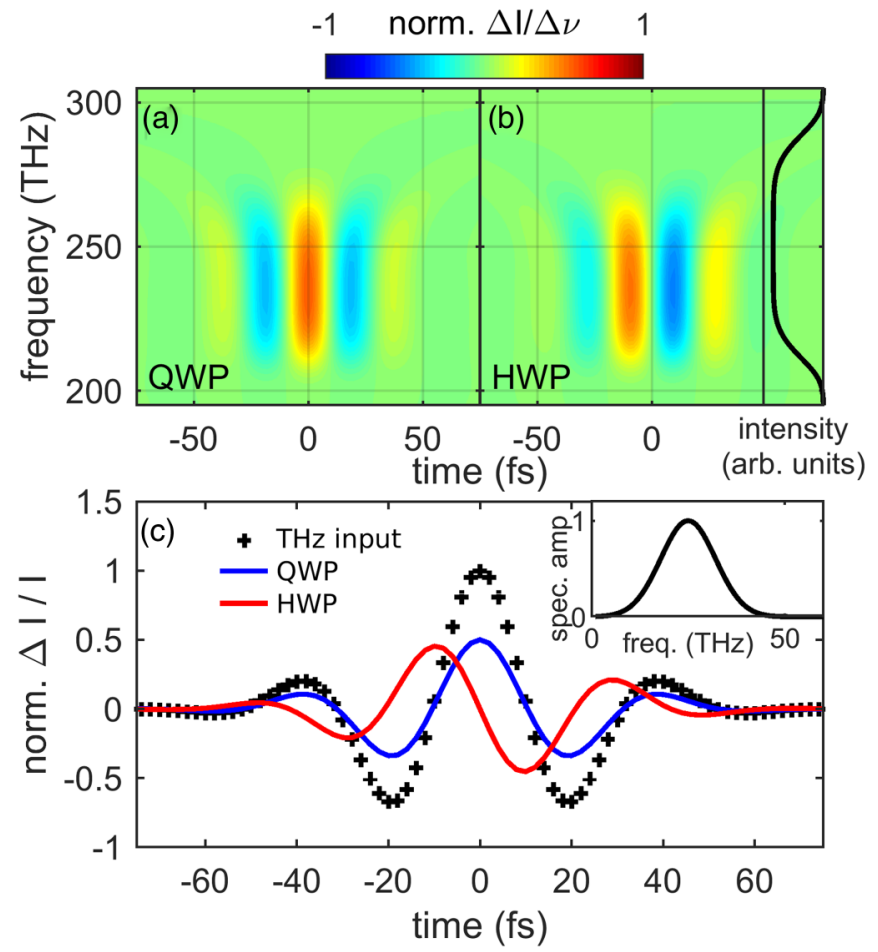

FIG. 9. Simulation results for quarter- and half-wave plate signals for given input spectra (black lines in insets) assuming $\varphi_{0}=0$ and only difference-frequency generation contributing. (a) Normalized differential currents measured using a QWP (color-coded) as a function of probe frequency and delay time. (b) Corresponding HWP signals depicted in the same manner. The inset on the right-hand side shows the probe spectrum. (c) QWP (dark blue) and HWP (light red) signals integrated over the full probe spectrum. The actual multiterahertz field is depicted by black crosses. The inset shows the normalized amplitude spectrum of the terahertz input.

field for comparison with electro-optic signals. Figures 9(a) and 9(b) show numerical results for $\varphi_{0}=0$ and a nonlinear interaction restricted to DFG. The high-frequency components of the probe spectrum (inset at right) contribute no effects, since DFG can only generate signals as an interference between downconverted and original field components. The transients for all frequencies are in phase for QWP and HWP measurements, respectively, but out of phase relative to each other. The spectrally integrated signals plotted in Fig. 9(c) are of equal peak amplitude with a relative phase shift of precisely $\pi / 2$. The QWP signal is in phase with the input multiterahertz electric field which is depicted by black crosses. Note that this finding holds only for $\varphi_{0}=0$. Inherent suppression of SFG is typically achieved by critically phase matching the DFG process in a uniaxial crystal. While this step allows use of a thicker EOX and therefore provides higher signal amplitudes, it also changes the delay-independent phase in a way that is hard to control quantitatively. Therefore, the phase relationship between electro-optic signals and the input electric field is not trivial in realistic experiments.

The situation is different when SFG and DFG contribute equally to the polarization changes in EOS. Figures 10(a) and 10(b) show spectrally resolved electro-optic signals from SFG and DFG, again with $\varphi_{0}=0$. The numerical results agree
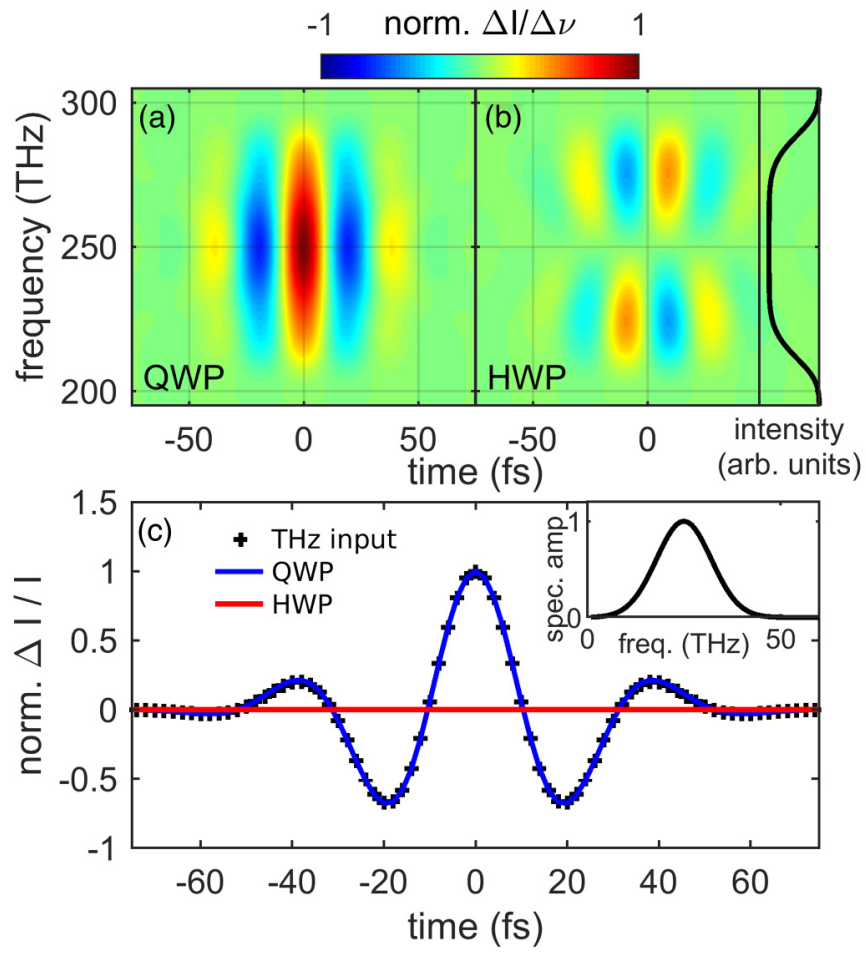

FIG. 10. Simulation results for quarter- and half-wave plate signals for given input spectra (black lines in insets) assuming a $\varphi_{0}=0$ and sum and difference-frequency generation contributing. (a) Normalized differential currents measured with a QWP (color-coded) as a function of probe frequency and delay time. (b) Corresponding HWP signals depicted in the same manner. (c) Spectrally integrated signals for QWP (dark blue) and HWP (light red). The actual multiterahertz field is depicted by black crosses and the inset shows the normalized amplitude spectrum of the terahertz input.

with the prediction from our intuitive picture in Fig. 3(b) and the experimental data acquired using ZnTe as an electro-optic detector [Figs. 6(a)-6(c) and Fig. 7]. The QWP signal is in phase over the entire probe spectrum, while the HWP signal inverts sign around the center frequency of the probe. This fact leads to integrated signals virtually identical with the input multiterahertz transient for the setting with a QWP [blue line in Fig. 10(c)] and a vanishing amplitude for the HWP (light red). To demonstrate that the concurrence between a normalized QWP signal and input field is independent of delay-independent phase in this case, we compute the signals in analogy to Fig. 10, but with $\varphi_{0}=-\pi / 4$. The spectrally resolved results are shown in Figs. 11(a) and 11(b). Here, the signals at the probe center frequency are identical, while the signals in the wings show opposite phase shifts for low- and high-frequency regions. Note that the direction of these shifts is in opposite directions between QWP and HWP. The spectrally integrated signals [blue and red dashed lines in Fig. 11(c)] are almost identical and in phase with the input field. Here the phase shifts occurring in the wings of the probe spectrum, where either DFG or SFG dominate, precisely compensate each other. This fact leads to a reduction in maximum amplitude as compared to the ideal scenario with $\varphi_{0}=0$ but not to a phase shift. Therefore, an experiment preserving the balance between SFG and DFG contributions 

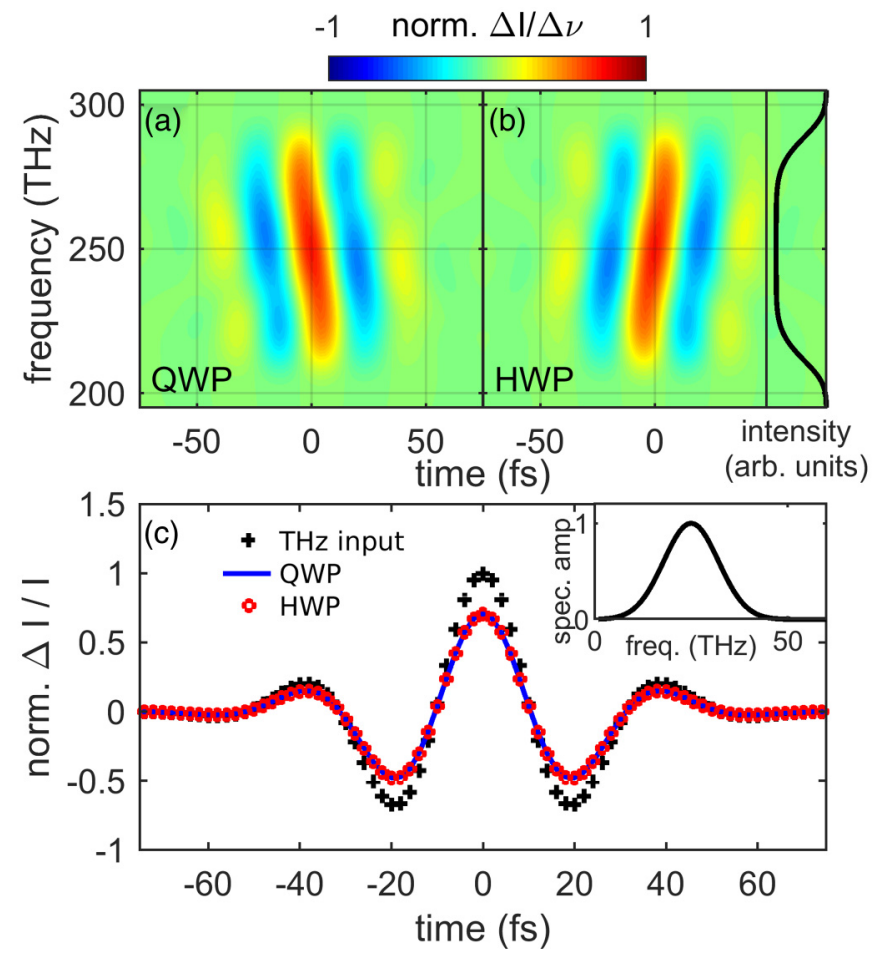

FIG. 11. Simulation results for quarter- and half-wave plate signals for given input spectra (black lines in insets) assuming $\varphi_{0}=$ $-\pi / 4$ and contributions from SFG and DFG. (a) Normalized differential photocurrents taken with QWP (color-coded) as a function of probe frequency and delay time. (b) Corresponding HWP signals depicted in the same manner. (c) Spectrally integrated signals for QWP (dark blue) and HWP (light red). The actual multiterahertz field is depicted by black crosses.

is ideal to reliably determine the precise carrier-envelope phase of the input field.

\section{F. Influence of the multiterahertz bandwidth}

So far, one of the crucial points was to explore how the accuracy of the absolute phases measured may or may not depend on the delay-independent phase. Therefore, we adopted a mid-infrared transient covering frequencies significantly below the total bandwidth of the probe. In this way, the comparison between multiterahertz input and electrooptic signals became as clear as possible. Now, the effect of different multiterahertz amplitude spectra is studied. For this purpose, we assume a broadband input spanning from 10 to $90 \mathrm{THz}$. Adding a quadratic phase leads to a chirped wave form with varying instantaneous frequency. In this way we can illustrate the effect of different multiterahertz spectra in one time trace. All other input parameters are kept identical with respect to the computations presented above and $\varphi_{0}=0$.

Calculations of the frequency-resolved differential photocurrent traces using a QWP or a HWP are color-coded in Figs. 12(a) and 12(b), respectively. The abscissa corresponds to the delay time $t_{D}$ along which the instantaneous frequency of the MIR input changes (see top axis). Relatively large multiterahertz frequencies are mixing with the probe spectrum at negative time delays. As long as the instantaneous input frequency exceeds $50 \mathrm{THz}$, minimum QWP and HWP signals are found in a region around the center of the probe spectrum. Again, an intuitive understanding of this fact may be gained from Fig. 4: the largest mid-infrared frequency that may interact with the probe in the center of its spectrum corresponds to half the probing bandwidth. If no smaller multiterahertz frequencies are available, no mixing to the probe center frequency can take place. For increasing time delays from -200 to $0 \mathrm{fs}$, lower multiterahertz frequencies start to contribute and the region without signal amplitude decreases in width until it completely vanishes for the QWP measurement [Fig. 12(a)]. At this point, the HWP signal amplitude still remains zero in the very center [Fig. 12(b)]. Here, SFG and DFG contribute in perfect antisymmetry and the polarization rotation cancels out. This situation corresponds to the sketch in Fig. 3(b). For $t_{D}>100 \mathrm{fs}$, the instantaneous MIR frequency drops below $40 \mathrm{THz}$. Therefore, a larger region of the probe spectrum receives equal contributions via sum and difference-frequency mixing, thus canceling the HWP signals in a larger region around the center of the probe spectrum in Fig. 12(b).

At the late delay times $t_{D}>150 \mathrm{fs}$ in Fig. 12, the situation corresponds to the expected behavior in the case of EOS for standard terahertz time-domain spectroscopy $(\mathrm{THz}$ TDS), where the bandwidth of the probe typically exceeds the maximum terahertz input frequency. Close to $t_{D}=0 \mathrm{fs}$, the conditions are comparable to the experimental data presented in this paper, where the multiterahertz input frequencies cover approximately half the probe bandwidth. At negative delay times $t_{D}$, a high-frequency MIR transient with multiple oscillations is sampled, as may be generated by DFG from two relatively narrowband NIR or visible pump spectra.

Now, we will examine the consequences of the changes of the instantaneous MIR frequency when filtering out specific parts of the probe spectrum. Figure 12(c) depicts the input electric field transient (black) together with its temporal derivative (light dotted green line). We first assume a low-pass filter in the center of the probe spectrum at $250 \mathrm{THz}$. The transients obtained when sampling with a QWP or HWP are shown as blue and red graphs, respectively. In contrast to the input transient, the envelopes of both HWP and QWP signals decrease for increasing input frequency at negative delay times, because detection of low frequencies is favored in this configuration. The corresponding spectral amplitudes are depicted in the inset with the same color coding: the amplitude spectrum of our chirped input field has a flat-top shape centered around $50 \mathrm{THz}$ (black line). When computing the temporal derivative of this transient, we obtain a blue-shifted spectrum peaked close to the high-frequency end at $80 \mathrm{THz}$ (green). In contrast, the maxima of both amplitude spectra for the QWP (dark blue) and HWP (dashed red line) signals are located close to the low-frequency cutoff at around $30 \mathrm{THz}$. The reason for the slightly different spectral peak positions is as follows: DFG dominates the mixing in the lower half of the probe spectrum which is detected here, but residual SFG close to the center frequency of the probe generates a HWP signal corresponding to low MIR frequencies, which contributes with opposite sign. Instead, both contributions add up in the QWP signal, effectively shifting its spectral amplitude to even lower frequencies [compare the discussion about response functions in Sec. IV D]. 


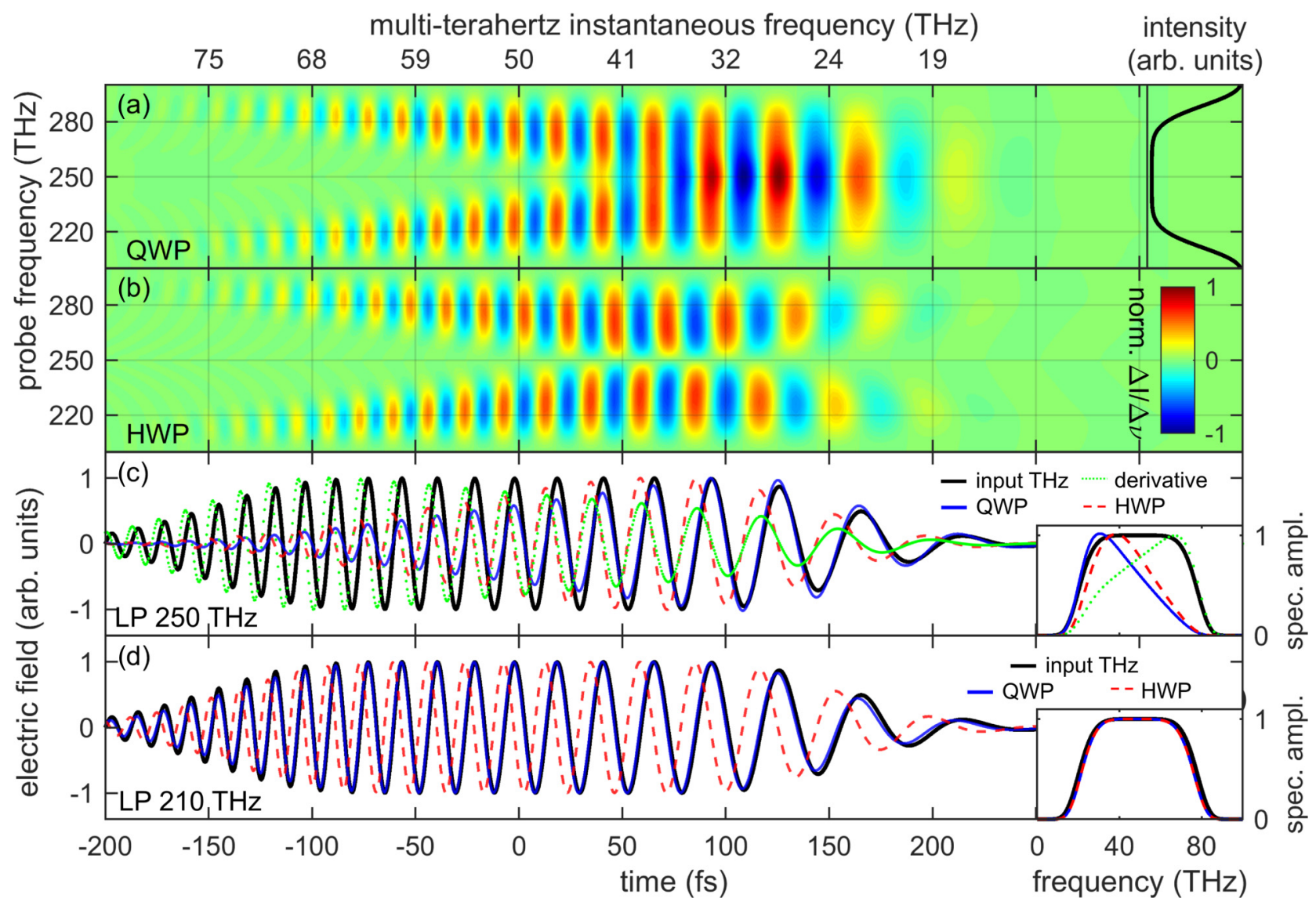

FIG. 12. Simulation results for a chirped multiterahertz transient sampled using QWP and HWP. (a) Color-coded differential photocurrents measured with a QWP as a function of probe frequency and delay time. The top axis shows the instantaneous frequency of the input multiterahertz field. (b) Color-coded differential photocurrents measured with a HWP as a function of probe frequency and delay time. The inset on the right-hand side illustrates the probe spectrum. (c), (d) The chirped input multiterahertz transient (black solid line) and its temporal derivative (light dotted green line) and the transients recorded by EOS with QWP (dark blue solid line) and HWP (dashed red line) employing low-pass filters with cut-off frequencies at 250 and $210 \mathrm{THz}$, respectively. The insets show the corresponding amplitude spectra.

In Fig. 12(d), we now discuss the situation for a low-pass filter at $210 \mathrm{THz}$ which is close to the low-frequency cutoff of the probe. In this setting, SFG contributions are maximally suppressed. The time trace obtained with a QWP (blue line) becomes virtually identical to the input electric field transient (black). Correspondingly, the two amplitude spectra in the inset at right sit almost perfectly on top of each other. The time trace calculated for a HWP [red line in Fig. 12(d)] exhibits the same peak amplitudes as the QWP signal, but it is phase shifted by precisely $\pi / 2$. As depicted in the right inset, also the amplitude spectrum (red dashed) is virtually identical to the input transient and the QWP signal. In total, we can summarize that spectral filtering close to the edges of the probe spectrum produces a time trace maximally close to the input electric field with a QWP and its Hilbert transform with a HWP. This situation is represented by the phasor picture in Fig. 3(a). Note that isolating the DFG or SFG contribution in this manner comes at the cost of a reduction in the overall signal-to-noise ratio of the measurement. Appropriate phase matching can mitigate this shortcoming. On the other hand, the static birefringence then introduces a finite $\varphi_{0}$ coming with an uncertainty about the phase relationship between measured transients and the actual input electric field, as discussed above.

\section{SUMMARY AND OUTLOOK}

In conclusion, we have demonstrated the measurement of the time-resolved electric field and its Hilbert transform both in critically phase-matched and isotropic nonlinear crystals. For this purpose, we augmented the typical configuration for electro-optic sampling by using quarter- and half-wave plates to detect the changes in both polarization ellipticity and rotation. These signals are spectrally resolved by bandpass filters inserted after the three-wave mixing step. The crucial parameters are identified to be the balance of sum and difference-frequency generation between multiterahertz and probe pulses and the delay-independent phase $\pi / 2+\varphi_{0}$ between the radiation generated by these processes and the original probe pulses. Additionally, we have introduced an intuitive picture that efficiently visualizes the synthesis of QWP and HWP signals.

We find that measurements employing a critically phasematched EOX favor SFG or DFG and yield a straightforward method to measure two transients which are Hilbert transforms of each other by using a QWP and HWP. In contrast, an isotropic EOX conserves the symmetry between SFG and DFG, leading to weak HWP signals when integrating over the full probe spectrum. Rigorous theoretical treatment and 
numerical simulations show that this symmetry renders electro-optic sampling with isotropic detectors robust against a finite $\varphi_{0}$. Spectral filtering in the low- or high-frequency side of the probe spectrum also breaks the symmetry between DFG and SFG. In the case of $\varphi_{0} \neq 0$, EOS exploiting predominantly one of these processes yields transients phase shifted against the input field. This effect is evident already from the intuitive model but quantified in theory and ultrabroadband experiments. The theoretical model also demonstrates that transients obtained by analyzing the polarization changes in the wings of the spectrum result in amplitude spectra almost identical to the input multiterahertz electric field. From all those findings, a clear strategy emerges for quantitative recording of the two quadratures of the electric field in the most accurate way and even simultaneously. In the future, our insights will be crucial for establishing a full quantum tomography of the electromagnetic field at subcycle time scales. To avoid divergences in a quantum description, fieldlike classical quantities which are well defined at singular points of time and space have to be replaced by amplitudes averaged over finite space-time volumes [37]. In our experiments, however, this aspect is automatically taken care of by the inherent need to work with a finite probe pulse duration and transverse mode area $[14,16]$.

\section{ACKNOWLEDGMENT}

Funding by the ERC (Advanced Grant No. 290876 "UltraPhase") is gratefully acknowledged.

\section{APPENDIX: CONSEQUENCES OF NONIDEAL PHASE RETARDERS AND INPUT POLARIZATION}

The main text discusses the fundamental principles dictating the connection between an input electric field transient and the induced polarization changes by the secondorder nonlinearity. They are assessed via balanced detection schemes including a quarter-wave plate to measure variations of the ellipticity or a half-wave plate to measure a polarization rotation. Our model predicts these signals are either in phase or phase shifted by $\pi / 2$. However, deviations from this behavior may occur because of imperfect fulfillment of the initial assumptions, that is, achromatic retardation of the wave plates as well as imperfect input polarization. The polarization may show finite ellipticity and/or a tilt against the principal axis of the Wollaston prism. Note that both deviations have the same consequences on the measurement process and we therefore discuss them together.

The limited precision of the wave plates is especially important since we are discussing ultrabroadband probe spectra, approaching a full octave. Although wave plates with a rather flat retardation in an even larger spectral range are available [38], they still exhibit a significant deviation of the retardation and rotation of the fast axis. The designs of such state-of-the-art components include about $4 \%$ deviation of the retardation and roughly $0.1^{\circ}$ variation of the fast and slow axes over the range of our probe spectrum. Additionally, the manufacturing precision of the overall thickness is limited to $\pm 3 \mathrm{~nm}$, potentially introducing even stronger deviations.
In terms of the model discussed in Sec. II, such imperfections mean that QWP and HWP signals are no longer represented by projections to precisely the imaginary and real axes in the complex plane, respectively. In principle, one may numerically evaluate Eq. (6) for each frequency with the specific retardation given by the datasheet of the wave plate to

(a)
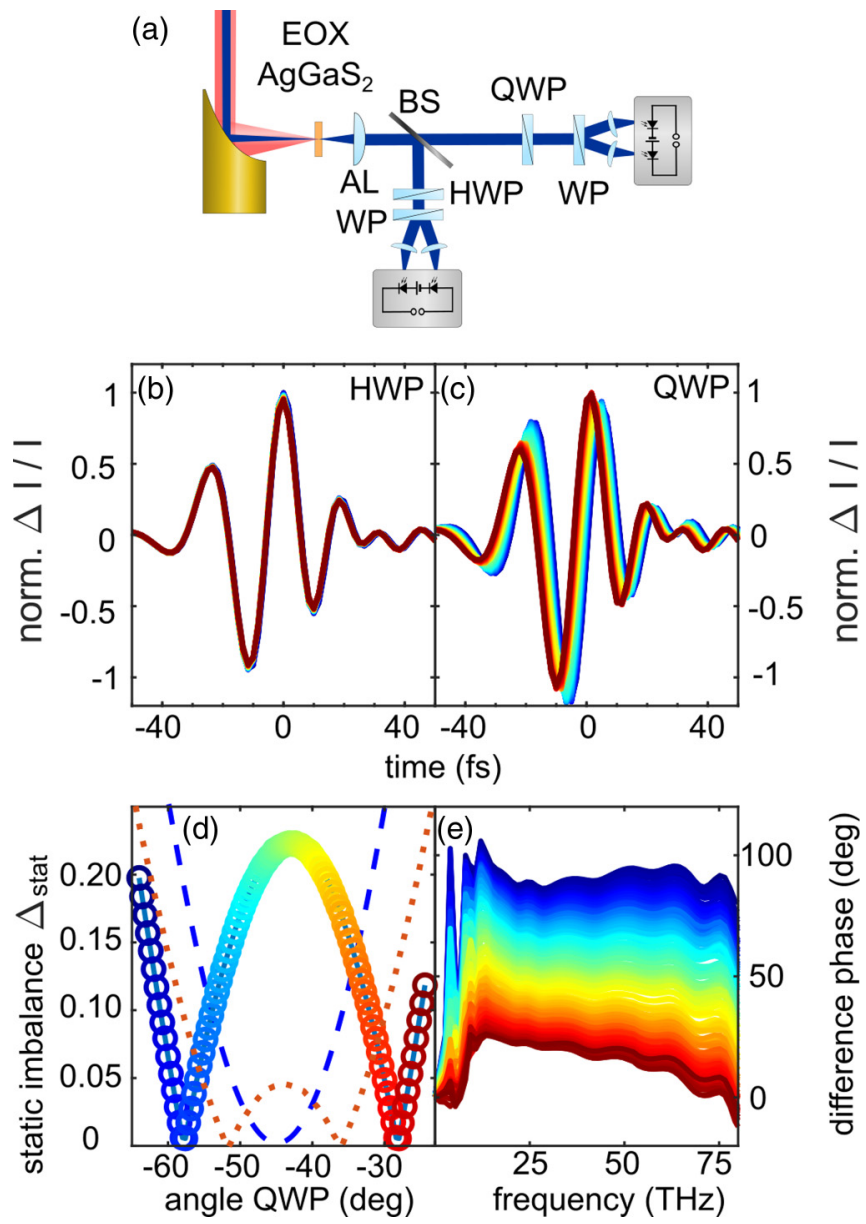

FIG. 13. (a) Experimental setup allowing the simultaneous detection of polarization rotation (using a half-wave plate) and ellipticity (using a quarter-wave plate) in EOS. After nonlinear mixing within an $\mathrm{AgGaS}_{2}$ crystal, an achromatic lens collimates the probe beam. A beam splitter (BS) consisting of a thin layer of Inconel on top of a silica substrate is used to separate the probe pulse trains. Afterwards, two balanced detection schemes with a QWP and a HWP, respectively, are implemented. (b), (c) Transients measured in the balanced detectors as a function of delay time in the HWP and QWP branches, respectively. The angle of the HWP is kept constant, while the angle of the QWP is varied over a range of almost $40^{\circ}$. (d) Static imbalance in the differential photocurrent due to angle of QWP with respect to the initial linear polarization axes of the probe. For a perfect QWP and perfect input polarization, a parabola touching the $x$ axis is expected (dashed blue line). The orange dotted line represents a typical measurement taken without the BS. The measured static imbalance is plotted as solid circles with a color code that corresponds to the QWP angle. (e) Difference phases between QWP and HWP measurements as a function of terahertz frequency for different QWP angles. The color coding of the graphs corresponds to the circles in panel (d). 
quantify deviations introduced to the electro-optic signals and static imbalances.

To experimentally explore such effects, we implement two balanced detection schemes that may be read out simultaneously [see Fig. 13(a)]. The probe pulse train is split after the EOX by means of a thin Inconel film (NiCrFe alloy, ratio: 75:16:9, thickness $28 \mathrm{~nm}$ ) on a fused-silica substrate. We repeat the measurement for several angles of the QWP with respect to the initial linear polarization of the probe, creating a static imbalance on the balanced photodetectors. The resulting transients are displayed in Fig. 13(b) (polarization rotation) and Fig. 13(c) (ellipticity). The QWP transients show significant changes in phase and amplitude, while the HWP transients are virtually identical. This fact demonstrates that during the measurement, no significant drift occurred and changes in the QWP transients are due only to the change of the orientation of the wave plate. Figure 13(d) describes the static imbalance $\Delta_{\text {stat }}$ between the two photocurrents in the balanced detector, which occurs independent of the multiterahertz field:

$$
\Delta_{\mathrm{stat}}=\left.\frac{I_{z}-I_{s}}{I_{z}+I_{s}}\right|_{E_{\mathrm{THz}}=0} .
$$

For a perfect QWP (or HWP) and input polarization, a parabola touching the $x$ axis at an angle of 45 degrees is expected (blue dashed line) [29]. The colored circles depict the static imbalance measured while rotating the QWP. The deviation from the parabola is explained by the limited achromaticity of the wave plate and the imperfect linear polarization. Static polarization changes may be introduced by the birefringence of the $\mathrm{AgGaS}_{2}$ sensor and the polarizationdependent reflection or transmission amplitudes and phases of the beam splitter. This situation is investigated by measuring the static imbalance in absence of the beam splitter (gray dashed line). These deviations have important consequences, since for ideal suppression of technical noise, it is necessary to precisely balance the two input channels of the BPD. Also note that the distribution of intensity fluctuations may not be similar to the distribution of intensity. Thus, the QWP angle that suppresses these fluctuations optimally might conserve a static imbalance introduced by the natural birefringence of the silver gallium sulfide.

Figure 13(e) shows the influence of the angle of the QWP on the difference phase between the QWP and HWP signals. Particularly, graphs depicted in a specific color correspond to an angle of the QWP where the circle in Fig. 13(d) is colored identically. Tuning the QWP over a range of $30^{\circ}$ changes the difference phase between both transients (compare Sec. III B) by almost $\pi / 2$, demonstrating that a misalignment of the QWP fast axis can lead to significant deviations in the carrier-envelope phase of the measured transient.

Note that the angle of the QWP for which the difference phase is $\pi / 2$ differs from the expected $-45^{\circ}$. This fact is due to the absorption in the Inconel layer. Such a beam splitter causes phase shifts in both transmission and reflection that may have significant dependence on the polarization, therefore modifying the delay-independent phase by adding a finite $\varphi_{0}$. Additionally, the reflection and transmission coefficients are dependent on the polarization, leading to further distortion of the polarization states. These aspects are not problematic here, since Fig. 13 only goes to demonstrate that misaligning the QWP might be necessary to balance the photocurrents and may introduce some deviations in the acquired signals. Consequently, dielectric beam splitters should be employed for simultaneous measurement of both quadratures, since they introduce only phase shifts of $\pi$.
[1] Q. Wu and X.-C. Zhang, Appl. Phys. Lett. 67, 3523 (1995).

[2] A. Nahata, A. S. Weling, and T. F. Heinz, Appl. Phys. Lett. 69, 2321 (1996).

[3] Q. Wu and X.-C. Zhang, Appl. Phys. Lett. 71, 1285 (1997).

[4] A. Leitenstorfer, S. Hunsche, J. Shah, M. C. Nuss, and W. H. Knox, Appl. Phys. Lett. 74, 1516 (1999).

[5] C. Kübler, R. Huber, S. Tübel, and A. Leitenstorfer, Appl. Phys. Lett. 85, 3360 (2004).

[6] A. Sell, R. Scheu, A. Leitenstorfer, and R. Huber, Appl. Phys. Lett. 93, 251107 (2008).

[7] S. Keiber, S. Sederberg, A. Schwarz, M. Trubetskov, V. Pervak, F. Krausz, and N. Karpowicz, Nat. Photonics 10, 159 (2016).

[8] R. Huber, F. Tauser, A. Brodschelm, M. Bichler, G. Abstreiter, and A. Leitenstorfer, Nature (London) 414, 286 (2001).

[9] J. Kröll, J. Darmo, S. S. Dhillon, X. Marcadet, M. Calligaro, C. Sirtori, and K. Unterrainer, Nature (London) 449, 698 (2007).

[10] K. W. Kim, A. Pashkin, H. Schäfer, M. Beyer, M. Porer, T. Wolf, C. Bernhard, J. Demsar, R. Huber, and A. Leitenstorfer, Nat. Mater. 11, 497 (2012).

[11] M. Först, C. Manzoni, S. Kaiser, Y. Tomioka, Y. Tokura, R. Merlin, and A. Cavalleri, Nat. Phys. 7, 854 (2011).

[12] T. Kampfrath, M. Battiato, P. Maldonado, G. Eilers, J. Nötzold, S. Mährlein, V. Zbarsky, F. Freimuth, Y. Mokrousov, S. Blügel,
M. Wolf, I. Radu, P. M. Oppeneer, and M. Münzenberg, Nat. Nanotechnol. 8, 256 (2013).

[13] O. Schubert, M. Hohenleutner, F. Langer, B. Urbanek, C. Lange, U. Huttner, D. Golde, T. Meier, M. Kira, S. W. Koch, and R. Huber, Nat. Photonics 8, 119 (2014).

[14] C. Riek, D. V. Seletskiy, A. S. Moskalenko, J. F. Schmidt, P. Krauspe, S. Eckart, S. Eggert, G. Burkard, and A. Leitenstorfer, Science 350, 420 (2015).

[15] A. S. Moskalenko, C. Riek, D. V. Seletskiy, G. Burkard, and A. Leitenstorfer, Phys. Rev. Lett. 115, 263601 (2015).

[16] C. Riek, P. Sulzer, M. Seeger, A. S. Moskalenko, G. Burkard, D. V. Seletskiy, and A. Leitenstorfer, Nature (London) 541, 376 (2017).

[17] I.-C. Benea-Chelmus, F. F. Settembrini, G. Scalari, and J. Faist, Nature (London) 568, 202 (2019).

[18] T. L. M. Guedes, M. Kizmann, D. V. Seletskiy, A. Leitenstorfer, G. Burkard, and A. S. Moskalenko, Phys. Rev. Lett. 122, 053604 (2019).

[19] M. Kizmann, T. L. de M. Guedes, D. V. Seletskiy, A. S. Moskalenko, A. Leitenstorfer, and G. Burkard, Nat. Phys. 15, 960 (2019).

[20] S. De Liberato, Phys. Rev. A 100, 031801(R) (2019).

[21] F. Lindel, R. Bennett, and S. Y. Buhmann, arXiv:1905.10200. 
[22] C. Silberhorn, Contemp. Phys. 48, 143 (2007).

[23] A. I. Lvovsky and M. G. Raymer, Rev. Mod. Phys. 81, 299 (2009).

[24] G. Leuchs, Contemp. Phys. 29, 299 (1988).

[25] S. Virally and B. Reulet, Phys. Rev. A 100, 023833 (2019).

[26] S. L. Hahn, Hilbert transforms, in The Transforms and Applications Handbook, edited by A. D. Poularikas (CRC Press, Boca Raton, FL, 1996), Chap. 7, pp. 463-629.

[27] G. Gallot and D. Grischkowsky, J. Opt. Soc. Am. B 16, 1204 (1999).

[28] D. J. Kane and R. Trebino, Opt. Lett. 18, 823 (1993).

[29] C. Riek, D. V. Seletskiy, and A. Leitenstorfer, Eur. J. Phys. 38, 024003 (2017).
[30] Q. Wu and X.-C. Zhang, Appl. Phys. Lett. 70, 1784 (1997).

[31] M. Born and E. Wolf, Principles of Optics: Electromagnetic Theory of Propagation, Interference and Diffraction of Light (Elsevier, New York, 2013).

[32] R. C. Jones, J. Opt. Soc. Am. 31, 488 (1941).

[33] P. S. Hauge, Surf. Sci. 56, 148 (1976).

[34] P. S. Hauge, Surf. Sci. 96, 108 (1980).

[35] K. Oguchi, N. Yasumatsu, and S. Watanabe, J. Opt. Soc. Am. B, 31, 3170 (2014).

[36] M. Porer, J.-M. Ménard, and R. Huber, Opt. Lett. 39, 2435 (2014).

[37] W. Heisenberg, The Physical Principles of the Quantum Theory (Dover, New York, 1949).

[38] J. Tinbergen, Astron. Astrophys. 23, 25 (1973). 